\title{
Phenomenology of SUSY with General Flavour Violation
}

\author{
Kamila Kowalska \\ National Centre for Nuclear Research, \\ Hoża 69, 00-681 Warsaw, Poland \\ E-mail: Kamila.Kowalska@fuw.edu.pl
}

Abstract: We discuss the consequences of relaxing the Minimal Flavour Violation assumption in the up-squark sector on the phenomenology of SUSY models. We study the impact of the off-diagonal entries in the soft SUSY-breaking matrices on the mass of the lightest Higgs scalar and we derive the approximate analytical formulae that quantify this effect. We show that $m_{h}$ can be enhanced by up to $13-14 \mathrm{GeV}$ in the case of the phenomenological MSSM with the inverted hierarchy of masses in the squark sector and zero stop mixing, and up to $4-5 \mathrm{GeV}$ in GUT-constrained scenarios where the magnitude of the enhancement is mitigated by renormalization group effects. We also perform a global analysis of an inverted hierarchy GFV scenario, taking into account the experimental bounds from the measurements of relic density, EW precision observables and $B$-physics. We show that the allowed parameter space of the model is strongly constrained by $m_{W}, \sin ^{2} \theta_{\text {eff }}$ and $\mathrm{BR}\left(B_{s} \rightarrow \mu^{+} \mu^{-}\right)$, requiring $m_{0}(3)<1500 \mathrm{GeV}$ and $m_{1 / 2}<1800 \mathrm{GeV}$, as well as a large non-zero $(2,3)$ entry in the up-squark trilinear matrix.

KEYWORDs: Supersymmetry Phenomenology

ARXIV EPRINT: 1406.0710 


\section{Contents}

1 Introduction 1

2 The Higgs boson mass in the GFV MSSM 3

3 Limits on $\left(\delta_{13}^{u}\right)_{L R},\left(\delta_{31}^{u}\right)_{L R},\left(\delta_{23}^{u}\right)_{L R}$ and $\left(\delta_{32}^{u}\right)_{L R} \quad 10$

4 GFV effects in GUT-constrained scenarios $\quad 14$

$\begin{array}{lll}5 & \text { Global analysis } & 18\end{array}$

6 Conclusions $\quad 22$

$\begin{array}{ll}\text { A Derivation of the formulae for } \Delta m_{h} & 23\end{array}$

\section{Introduction}

The discovery of the Higgs boson [1,2] was an unquestionable and historical success of the LHC $8 \mathrm{TeV}$ run. With a mass of around $126 \mathrm{GeV}$ [3], the new scalar is consistent with the predictions of both the Standard Model (SM) and its minimal supersymmetric extension. While in the former case the Higgs mass remains a free parameter of the theory, in the latter it is totally determined by the gauge and soft supersymmetry-breaking (SSB) sectors. In the Minimal Supersymmetric Standard Model (MSSM) the enhancement from the tree-level value, necessary to obtain $m_{h}$ in agreement with the experimental data, can be achieved through radiative corrections to the scalar potential. Those, to be large enough, require either relatively heavy stops or almost maximal mixing in the stop sector [4].

The phenomenology of the SUSY landscape after the Higgs discovery has been widely studied in the literature, both in the context of GUT-constrained scenarios, as well as of models described by a set of supersymmetric parameters defined at the electro-weak symmetry breaking (EWSB) scale (for a non-comprehensive list of articles see for example [5-14]). The vast majority of those analyses were performed under the assumption of Minimal Flavour Violation (MFV). In this framework the only source of flavour mixing in the sfermion sector are the CKM and PMNS matrices, so the supersymmetric contributions the the Flavour Changing Neutral Currents (FCNC) transitions are suppressed via the super-GIM mechanism, in analogy to what happens in the Standard Model. The MFV assumption can be realised in two different ways: a) the trilinear terms and soft masses are aligned with the corresponding Yukawa matrices, thus becoming nearly diagonal after rotation to the SCKM-basis; b) diagonal entries of soft mass matrices are degenerate so that the off-diagonal elements in the SCKM-basis are automatically zero. Moreover, if sfermions are heavy enough, SUSY loop contributions to the FCNC will be naturally 
suppressed regardless the SSB structure. The last assumption, combined with the nonobservation of SUSY particles at the LHC and the fact that the third generation of colour sfermions should not be too heavy in order to keep the fine tuning of the model reasonably low $[15,16]$, seems to somehow favour the pattern of so-called inverted hierarchy (IH) [17], where the first two generations of sfermions are significantly heavier than the third one.

However, in the most general case, the soft masses and trilinear terms do not need to be constrained in any way and can be treated as additional free parameters of the model. This General Flavour Violation (GVF) is a source of what is called the FCNC supersymmetric problem, as the unrestricted off-diagonal entries of SSB matrices can lead to disastrously large SUSY contributions to FCNC processes. Note that even in a relatively simple case when the soft masses and trilinears are diagonal in the interaction basis though not proportional to the unity matrix, after the rotation of the fermion fields to the physical basis the non-diagonal SSB terms arise, proportional to the mass splitting between the diagonal entries. This effect is particularly important in the case of the soft mass $\left(m_{\tilde{Q}}^{2}\right)_{i j}$, where the mass splitting impact can be enhanced by the corresponding CKM matrix elements.

The FCNC processes are not the only area where a possible discrepancy between the GFV structure of the soft-SUSY breaking sector and the experimental data can manifest. Firstly, it was observed in ref. [18] that the one-loop flavour violating corrections to the mass of the $W$ boson and the value of $\sin ^{2} \theta_{\text {eff }}$ can be very large and therefore provide severe limits on the allowed parameter space of a supersymmetric model. Secondly, the authors of ref. [19] showed that the annihilation cross-section of the neutralino can be enhanced if the squark mass splitting is increased by GFV effects, and that new annihilation channels can open due to the presence of a flavour-mixing coupling between squarks and neutralinos. Finally, also the Higgs sector can be affected by the presence of GFV soft SUSY-breaking terms. It was shown in refs. [18, 20] (recently updated in [21]) that the corresponding radiative corrections to the lightest $\mathrm{CP}$-even scalar mass due to flavour mixings between the second and third squark generation can be either moderate and positive (up to $2-4 \mathrm{GeV}$ ) or large and negative, leading to a reduction of $m_{h}$ well below the LEP limit. The latter effect was also observed in [22].

In this study we pursue the question as to what extent the Higgs boson mass can be actually enhanced by the non-diagonal SSB terms without introducing heavy stops or maximal stop mixing. We derive approximate formulae that allow to quantify this effect analytically. We then show that the strongest Higgs mass enhancement can be achieved through non-zero $(2,3),(3,2),(1,3)$ and $(3,1)$ entries of the up-squark trilinear coupling for a particular choice of SSB mass matrices, namely in an inverted hierarchy scenario where the first two generations of sfermions are significantly heavier than the third one. The size of the GFV contribution can reach $13-14 \mathrm{GeV}$ in the case of the phenomenological MSSM, and up to $3-5 \mathrm{GeV}$ for the hierarchical model defined at the GUT scale. We also show that the enhancement in $m_{h}$ can be significantly reduced by introducing non-zero stop mixing.

We also analyse the consistency of a GUT-constrained inverted hierarchy scenario with other experimental data, in particular the measurement of relic density by PLANCK [23], constraints from B-physics and EW precision observables [24]. We emphasise that the latter set of constraints is of key importance as GFV effects due to mass splitting between the 
first/second and third generation give rise to large one-loop corrections to $m_{W}$ and $\sin ^{2} \theta_{\text {eff }}$. As a consequence, a part of the parameter space corresponding to the universal mass of the third generation squarks heavier than $1500 \mathrm{GeV}$ is strongly disfavoured. Finally, we point out a tension between the measurement of $\mathrm{BR}\left(\mathrm{B}_{\mathrm{s}} \rightarrow \mu^{+} \mu^{-}\right)$and the GFV assumption, which can be significantly reduced if a large non-zero $(2,3)$ entry in the up-squark trilinear matrix is present.

This paper is organised as follows. In section 2 we discuss the enhancement of the Higgs boson mass due to GFV effects and we derive approximate formulae to quantify their impact. In section 3 we calculate the limits from the FCNC processes, renormalization of the CKM matrix, and vacuum stability on the relevant GFV parameters. In section 4 we analyse the corresponding effects in the models defined at the GUT scale and show that the Higgs mass enhancement is reduced due to the effects of RGEs. In section 5 we present the results of a global analysis that combines GFV effects in the Higgs sector with other experimental data. We summarise our findings in section 6 .

\section{The Higgs boson mass in the GFV MSSM}

We will start this section with a brief review of the notation we will be using throughout the paper. In the interaction basis, the R-parity conserving superpotential of the MSSM is given by

$$
W=\epsilon_{a b}\left[H_{2}^{b} Q_{i}^{a}\left(Y_{u}\right)_{i j} \bar{U}_{j}+H_{1}^{b} Q_{i}^{a}\left(Y_{d}\right)_{i j} \bar{D}_{j}+H_{1}^{b} L_{i}^{a}\left(Y_{e}\right)_{i j} \bar{E}_{j}-\mu H_{1}^{a} H_{2}^{b}\right]
$$

where $a, b=1,2$ indicate $\mathrm{SU}(2)$ indices and $i, j=1,2,3$ are generation indices. The soft SUSY-breaking part of the lagrangian can be written as

$$
\begin{aligned}
\mathcal{L}_{\text {soft }}= & \tilde{Q}_{i}^{\dagger}\left(m_{\tilde{Q}}^{2}\right)_{i j} \tilde{Q}_{j}+\tilde{U}_{i}^{\dagger}\left(m_{\tilde{u}}^{2}\right)_{i j} \tilde{U}_{j}+\tilde{D}_{i}^{\dagger}\left(m_{\tilde{d}}^{2}\right)_{i j} \tilde{D}_{j}+\tilde{L}_{i}^{\dagger} m_{\tilde{L}_{i j}} \tilde{L}_{j}+\tilde{E}_{i}^{\dagger} m_{\tilde{e}_{i j}} \tilde{E}_{j}+, \\
& +m_{H_{d}}^{2} H_{1}^{*} H_{2}+m_{H_{u}}^{2} H_{2}^{*} H_{2}+\left(m_{3}^{2} \epsilon_{a b} H_{1}^{a} H_{2}^{b}+h . c .\right) \\
& +\epsilon_{a b}\left(\left(T_{u}\right)_{i j} H_{2}^{b} \tilde{Q}_{i}^{a} \tilde{U}_{j}^{*}+\left(T_{d}\right)_{i j} H_{1}^{b} \tilde{Q}_{i}^{a} \tilde{D}_{j}^{*}+\left(T_{e}\right)_{i j} H_{1}^{b} \tilde{L}_{i}^{a} \tilde{E}_{j}^{*}\right)+\text { h.c.. }
\end{aligned}
$$

The rotation of the quark fields into the basis in which they are diagonal - the so called super-CKM basis - means that also the squarks need to be rotated accordingly (since in this paper we are only interested in the effects coming from the inter-generation mixing in the quark/squark sector, we will treat neutrinos as massless, henceforth assuming that there is no tree-level mixing between the leptons). Defining the quark rotation matrices $V$ as

$$
Q_{\mathrm{sCKM}}=\left(\begin{array}{c}
V_{L}^{u} U \\
V_{L}^{d} D
\end{array}\right), \quad \bar{U}_{\mathrm{sCKM}}=V_{R}^{u} \bar{U}, \quad \bar{D}_{\mathrm{sCKM}}=V_{R}^{d} \bar{D}
$$

and demanding them to diagonalise the Yukawa matrices

$$
Y_{u}^{\text {diag }}=V_{R}^{u} Y_{u}^{T} V_{L}^{u \dagger}, \quad Y_{d}^{\text {diag }}=V_{R}^{d} Y_{d}^{T} V_{L}^{d \dagger},
$$


the soft SUSY-breaking mass matrices and the trilinear terms in the super-CKM basis take the form:

$$
\begin{array}{lll}
\left(m_{\tilde{Q}}^{2}\right)_{L L}=V_{L}^{d} m_{\tilde{Q}}^{2} V_{L}^{d \dagger}, & \left(m_{\tilde{U}}^{2}\right)_{R R}=V_{R}^{u} m_{\tilde{u}}^{2} V_{R}^{u \dagger}, & \left(m_{\tilde{D}}^{2}\right)_{R R}=V_{R}^{d} m_{\tilde{d}}^{2} V_{R}^{d \dagger}, \\
\left(m_{\tilde{U}}^{2}\right)_{L R}=\frac{v_{2}}{\sqrt{2}} V_{R}^{u}\left(T_{u}\right) V_{L}^{u \dagger}, & \left(m_{\tilde{D}}^{2}\right)_{L R}=\frac{v_{1}}{\sqrt{2}} V_{R}^{d}\left(T_{d}\right) V_{L}^{d \dagger} . &
\end{array}
$$

The $6 \times 6$ mass matrices for the up and down squarks are then constructed as (we follow here the notation of the SLHA2 [25]):

$$
\begin{aligned}
& \mathcal{M}_{\tilde{u}}^{2}=\left(\begin{array}{cc}
K\left(m_{\tilde{Q}}^{2}\right)_{L L} K^{\dagger}+\left(m_{u}^{\text {diag }}\right)^{2}-\frac{\cos 2 \beta}{6}\left(m_{Z}^{2}-4 m_{W}^{2}\right) \mathbb{I} & \left(m_{\tilde{U}}^{2}\right)_{L R}^{\dagger}-m_{u}^{\text {diag }} \mu^{*} \cot \beta \\
\left(m_{\tilde{U}}^{2}\right)_{L R}-m_{u}^{\operatorname{diag}} \mu \cot \beta & \left(m_{\tilde{U}}^{2}\right)_{R R}+\left(m_{u}^{\text {diag }}\right)^{2}+\frac{2 \cos 2 \beta}{3} m_{Z}^{2} \sin ^{2} \theta_{W} \mathbb{I}
\end{array}\right), \\
& \mathcal{M}_{\tilde{d}}^{2}=\left(\begin{array}{cc}
\left(m_{\tilde{Q}}^{2}\right)_{L L}+\left(m_{d}^{\text {diag }}\right)^{2}-\frac{\cos 2 \beta}{6}\left(m_{Z}^{2}+2 m_{W}^{2}\right) \mathbb{I} & \left(m_{\tilde{D}}^{2}\right)_{L R}^{\dagger}-m_{d}^{\text {diag }} \mu^{*} \tan \beta \\
\left(m_{\tilde{D}}^{2}\right)_{L R}-m_{d}^{\operatorname{diag}} \mu \tan \beta & \left(m_{\tilde{D}}^{2}\right)_{R R}+\left(m_{d}^{\text {diag }}\right)^{2}-\frac{\cos 2 \beta}{3} m_{Z}^{2} \sin ^{2} \theta_{W} \mathbb{I}
\end{array}\right) .
\end{aligned}
$$

In the above $m_{u}^{\text {diag }}=\frac{v_{2}}{\sqrt{2}} Y_{u}^{\text {diag }}$ and $m_{d}^{\text {diag }}=\frac{v_{1}}{\sqrt{2}} Y_{d}^{\text {diag }}$ are the diagonal matrices of quark masses, $\theta_{W}$ is the Weinberg angle, $\tan \beta$ is the ratio of the Higgs doublets' vacuum expectation values $(\mathrm{VEV}), \tan \beta \equiv \frac{v_{2}}{v_{1}}$, and $\mathbb{I}$ denotes the unity matrix in the generation space. The CKM matrix is defined as $K=V_{L}^{u \dagger} V_{L}^{d}$. Note that the left-handed blocks in $\mathcal{M}_{\tilde{u}}^{2}$ and $\mathcal{M}_{\tilde{d}}^{2}$ can not be simultaneously diagonal in the SCKM-basis if any mass splitting in $\left(m_{Q}^{2}\right)_{L L}$ is present. That is an important issue and we will come back to it in section 5 .

It is convenient to parametrise the non-diagonal entries of the squark mass matrices given in eq. (2.5) in terms of dimensionless parameters $\left(\delta_{i j}\right)_{A B}$, normalised to the geometrical average of the diagonal elements:

$$
\left(\delta_{i j}^{u}\right)_{A B}=\frac{\left(m_{\tilde{U}}^{2}\right)_{A B}^{i j}}{\sqrt{\left(m_{\tilde{U}}^{2}\right)_{B B}^{i i}\left(m_{\tilde{U}}^{2}\right)_{A A}^{j j}}}, \quad\left(\delta_{i j}^{d}\right)_{A B}=\frac{\left(m_{\tilde{D}}^{2}\right)_{A B}^{i j}}{\sqrt{\left(m_{\tilde{D}}^{2}\right)_{B B}^{i i}\left(m_{\tilde{D}}^{2}\right)_{A A}^{j j}}},
$$

where for consistency we defined $\left(m_{\tilde{U}}^{2}\right)_{L L}=K\left(m_{\tilde{Q}}^{2}\right)_{L L} K^{\dagger}$ and $\left(m_{\tilde{D}}^{2}\right)_{L L}=\left(m_{\tilde{Q}}^{2}\right)_{L L}$.

We can now proceed to discuss the dependence of the lightest Higgs boson mass on the size of parameters $\left(\delta_{i j}\right)_{A B}$. The full one-loop corrections to the masses of the Higgs scalars in the GFV framework have been calculated by several groups in the diagrammatic approach and implemented in the publicly available numerical codes, FeynHiggs [26-29] and SPheno [30,31]. In the numerical analysis throughout the paper we will use SPheno_v.3.2.4.

To have a grasp of possible effects that can arise after taking into account the non-zero values of parameters $\left(\delta_{i j}\right)_{A B}$, we will start with deriving approximate analytical formulae for the GFV corrections to the Higgs boson mass. In what follows we adopt the procedure proposed in section 6 of ref. [32], based on the effective potential technique [33, 34], as well as the subsequent results obtained in ref. [4] where the effects of the mixing and non-degeneracy of the soft masses in the stop sector have been thoroughly analysed.

In order to derive relatively simple expressions that would parametrise the GFV effects, in the following we will limit ourselves to analysing the contribution from the up-squark sector only, which is known to be the dominating one. ${ }^{1}$ Let us further assume that the

\footnotetext{
${ }^{1}$ In principle the contributions from the bottom and tau sectors can become significant for large $\tan \beta$. However, by choosing $A_{b, \tau}=0$ and small $\mu$ their impact can be strongly reduced.
} 
diagonal elements of the up squark mass matrix $\mathcal{M}_{\tilde{u}}^{2}$ are degenerate and equal to the common mass $\tilde{m}^{2}$. We also neglect the terms of the order of $m_{Z}^{2}$ and all the quarks masses but $m_{t}$. The matrix (2.6) takes then the form:

$$
\mathcal{M}_{\tilde{u}}^{2}=\tilde{m}^{2}\left(\begin{array}{ccc|ccc}
1 & \left(\delta_{12}^{u}\right)_{L L} & \left(\delta_{13}^{u}\right)_{L L} & 0 & \left(\delta_{21}^{u}\right)_{L R} & \left(\delta_{31}^{u}\right)_{L R} \\
\left(\delta_{12}^{u}\right)_{L L} & 1 & \left(\delta_{23}^{u}\right)_{L L} & \left(\delta_{12}^{u}\right)_{L R} & 0 & \left(\delta_{32}^{u}\right)_{L R} \\
\left(\delta_{13}^{u}\right)_{L L} & \left(\delta_{23}^{u}\right)_{L L} & 1+\frac{m_{t}^{2}}{\tilde{m}^{2}} & \left(\delta_{13}^{u}\right)_{L R} & \left(\delta_{23}^{u}\right)_{L R} & \frac{\left\langle v_{2}\right\rangle \tilde{X}_{t}}{\sqrt{2} \tilde{m}^{2}} \\
\hline 0 & \left(\delta_{12}^{u}\right)_{L R} & \left(\delta_{13}^{u}\right)_{L R} & 1 & \left(\delta_{12}^{u}\right)_{R R} & \left(\delta_{13}^{u}\right)_{R R} \\
\left(\delta_{21}^{u}\right)_{L R} & 0 & \left(\delta_{23}^{u}\right)_{L R} & \left(\delta_{12}^{u}\right)_{R R} & 1 & \left(\delta_{23}^{u}\right)_{R R} \\
\left(\delta_{31}^{u}\right)_{L R} & \left(\delta_{32}^{u}\right)_{L R} & \frac{\left\langle v_{2}\right\rangle \tilde{X}_{t}}{\sqrt{2} \tilde{m}^{2}} & \left(\delta_{13}^{u}\right)_{R R}\left(\delta_{23}^{u}\right)_{R R} & 1+\frac{m_{t}^{2}}{\tilde{m}^{2}}
\end{array}\right),
$$

where we defined $\tilde{X}_{t}=\left(T_{u}\right)_{33}-Y_{t} \mu \cot \beta$ in analogy the the common mixing parameter $X_{t}$. Note that the MFV case corresponds to $\left(\delta_{i j}\right)_{A B}=0$.

We will now discuss the GFV corrections to the Higgs boson mass, assuming various structures of the mass matrix (2.8). Here we present only the final results. The details of calculation can be found in appendix A.

Case 1: no GFV and $\tilde{\boldsymbol{X}}_{\boldsymbol{t}} \neq \mathbf{0}$. We will start with the well known scenario of nonzero mixing in the stop sector, which we will use as a reference case while studying the GFV effects. The masses of physical stops are given by $\tilde{m}_{t_{1}}^{2}=\tilde{m}^{2}+m_{t}^{2}-\tilde{X}_{t} \frac{v_{2}}{\sqrt{2}}, \tilde{m}_{t_{2}}^{2}=$ $\tilde{m}^{2}+m_{t}^{2}+\tilde{X}_{t} \frac{v_{2}}{\sqrt{2}}$. The one-loop correction to $m_{h}$ reads:

$$
\Delta m_{h}^{2}=\frac{3}{8 \pi^{2} v^{2}} Y_{t}^{4} v_{2}^{4} \ln \frac{\tilde{m}^{2}}{m_{t}^{2}}+\frac{3 v_{2}^{4}}{8 \pi^{2} v^{2}}\left[\frac{\tilde{X}_{t}^{2}}{\tilde{m}^{2}}\left(Y_{t}^{2}-\frac{\tilde{X}_{t}^{2}}{12 \tilde{m}^{2}}\right)\right] .
$$

If the trilinear term $T_{33}^{u}$ is proportional to the top Yukawa coupling, $T_{33}^{u}=Y_{t} A_{33}^{u}$, the above expression takes the usual form given in [4].

Case 2: $\left(\delta_{23}^{u}\right)_{L R} \neq 0$ or $\left(\delta_{13}^{u}\right)_{L R} \neq 0$. The presence of non-zero off-diagonal term $\left(\delta_{23}^{u}\right)_{L R}$ in the mass matrix $\left(m_{\tilde{U}}^{2}\right)_{L R}$ induces chirality flipping mixing between stop $\tilde{t}_{L}$ and scharm $\tilde{c}_{R}$, leading to the following mass eigenstates: $m_{\tilde{t}_{R}}^{2}=m_{t}^{2}+\tilde{m}^{2}, m_{\tilde{c}_{L}}^{2}=\tilde{m}^{2}$, $m_{1}^{2}=\frac{1}{2} m_{t}^{2}+\tilde{m}^{2}-\frac{1}{2} \sqrt{m_{t}^{4}+4 \tilde{m}^{4}\left(\delta_{23}^{u}\right)_{L R}^{2}}, m_{2}^{2}=\frac{1}{2} m_{t}^{2}+\tilde{m}^{2}+\frac{1}{2} \sqrt{m_{t}^{4}+4 \tilde{m}^{4}\left(\delta_{23}^{u}\right)_{L R}^{2}}$, where we assumed that $\tilde{X}_{t}=0$. Exactely the same effect arises in the case of $\tilde{u}_{R}-\tilde{t}_{L}$ mixing, with $\left(\delta_{23}^{u}\right)_{L R}$ replaced by $\left(\delta_{13}^{u}\right)_{L R}$.

If $\left(\delta_{23}^{u}\right)_{L R}>\frac{m_{t}^{2}}{2 \tilde{m}^{2}}$ (which corresponds to $\left(\delta_{23}^{u}\right)_{L R}>0.06$ for $\left.\tilde{m}=0.5 \mathrm{TeV}\right)$ the masses of the mixed eigenstates reduce to $m_{\tilde{t}_{1}}^{2}=\tilde{m}^{2}+\frac{1}{2} m_{t}^{2}+\tilde{m}^{2}\left(\delta_{23}^{u}\right)_{L R}, m_{\tilde{c}_{1}}^{2}=\tilde{m}^{2}+\frac{1}{2} m_{t}^{2}-\tilde{m}^{2}\left(\delta_{23}^{u}\right)_{L R}$. Since $\left(\delta_{23}^{u}\right)_{L R}$ depends linearly on the VEV $v_{2}$ (see eq. (2.5)), its correction to the Higgs boson mass is similar to the one generated by the stop mixing term $\tilde{X}_{t}$,

$$
\Delta m_{h}^{2}\left(\left(\delta_{23}^{u}\right)_{L R}\right)=\frac{3}{4 \pi^{2}}\left[\tilde{m}^{2}\left(\delta_{23}^{u}\right)_{L R}^{2}\left(\frac{1}{2} Y_{t}^{2} \sin ^{2} \beta-\frac{\tilde{m}^{2}\left(\delta_{23}^{u}\right)_{L R}^{2}}{6 v^{2}}\right)\right] .
$$

Its is straightforward to check that the maximal effect is expected when $\left(\delta_{23}^{u}\right)_{L R} \simeq$ $\sqrt{3 / 2} v Y_{t} \sin \beta / \tilde{m}$. 


\begin{tabular}{|c|c|c|c|c|c|c|c|c|c|c|}
\hline & $M_{1}$ & $M_{2}$ & $M_{3}$ & $A_{t, b, \tau}$ & $\mu$ & $m_{A}$ & $\tan \beta$ & $m_{\tilde{L}, \tilde{e}}$ & $m_{\tilde{Q}_{1,2}, \tilde{u}_{1,2}, \tilde{d}}$ & $m_{\tilde{Q}_{3}, \tilde{u}_{3}}$ \\
\hline BP1 & 126 & 233 & 670 & 0 & 242 & 1860 & 20 & 5950 & 560 & 530 \\
BP2 & 126 & 233 & 670 & 0 & 362 & 1712 & 30 & 1455 & 945 & 850 \\
BP3 & 126 & 233 & 670 & 0 & 462 & 1512 & 30 & 2500 & 1300 & 1200 \\
\hline
\end{tabular}

Table 1. Input parameters for benchmark points BP1 - BP3 at $M_{\mathrm{SUSY}}$. The masses and trilinear terms are in $\mathrm{GeV}$.

Case 3: $\left(\delta_{12}^{u}\right)_{L R} \neq \mathbf{0}$. The presence of this term induces the mixing between $\tilde{u}_{R}$ and $\tilde{c}_{L}$, while the stops remain degenerate. The masses of the mixed eigenstates are given by $m_{1}^{2}=$ $\tilde{m}^{2}+\tilde{m}^{2}\left(\delta_{12}^{u}\right)_{L R}$ and $m_{2}^{2}=\tilde{m}^{2}-\tilde{m}^{2}\left(\delta_{12}^{u}\right)_{L R}$. The terms proportional to $v_{2}^{2}$ are suppressed by the corresponding Yukawa couplings and have been neglected. On the other hand, since at this point we do not make any assumption about the size of the non-diagonal term $\left(\delta_{12}^{u}\right)_{L R}$, its contribution to the Higgs boson mass should be taken into account and gives:

$$
\Delta m_{h}^{2}\left(\left(\delta_{12}^{u}\right)_{L R}\right)=-\frac{3}{24 \pi^{2} v^{2}} \tilde{m}^{4}\left(\delta_{12}^{u}\right)_{L R}^{4}
$$

Notice that, unlike the case of $\left(\delta_{13}^{u}\right)_{L R}$ or $\left(\delta_{23}^{u}\right)_{L R}$ driven mixing, this contribution is always negative.

To illustrate the above discussion, we calculated the GFV-corrected Higgs boson mass with SPheno_v.3.2.4 and compared it with the analytical formulae given in eq. (2.10) and eq. (2.11). Our three benchmark points at the scale $M_{\mathrm{SUSY}}=\sqrt{\tilde{m}_{t_{1}} \tilde{m}_{t_{2}}}$ are defined in table 1. Gaugino masses, $\mu$ and trilinear terms were chosen to suppress the corrections not related to parameters $\left(\delta^{u}\right)_{L R}$, in particular those induced by mixing in the sbottom sector. ${ }^{2}$ The results are presented in figure 1 . The thin dashed lines indicate the prediction of the approximate one-loop formulae, while the solid lines show the output from SPheno. One observes that $m_{h}$ can be enhanced through the GFV contribution by up to $3 \mathrm{GeV}$. We confirm here the results first obtained in refs. [20] and [21] where a distinctive "M-shape" Higgs mass dependence on $\left(\delta_{23}^{u}\right)_{L R}$ and $\left(\delta_{32}^{u}\right)_{L R}$ was presented.

Finally, it is worth to make one more remark. An explicit proportionality of $\Delta m_{h}^{2}$ to the common supersymmetric mass scale $\tilde{m}$ in eqs. (2.10) and (2.11) is not in contradiction with an expected decoupling property of non-logarithmic finite corrections to the Higgs boson mass. One should keep in mind that the parameters $\left(\delta_{i j}\right)_{L R}$, defined in eq. (2.7), scale as $1 / \tilde{m}^{2}$ when the off-diagonal trilinear terms $T_{i j}^{u}$ are fixed, so the GFV corrections indeed decouple when $\tilde{m}$ increases. As a confirmation, in figure $1(\mathrm{c})$ we show a dependence of $\Delta m_{h}^{2}$ on the common SUSY scale for the benchmark points defined in table 1 . The results were obtained under the assumption that flavour-violating entries $T_{23}^{u}$ were fixed at the values allowing for the maximal Higgs boson mass enhancement. As in the previous plots, the thin dashed lines indicate the prediction of the approximate one-loop formulae, while the solid lines show the output from SPheno. In both cases a clear decoupling behaviour of the GFV correction can be observed.

\footnotetext{
${ }^{2}$ The corresponding corrections to the Higgs boson mass squared, calculated using the formulae given in [4], do not exceed $1.5 \mathrm{GeV}^{2}$.
} 


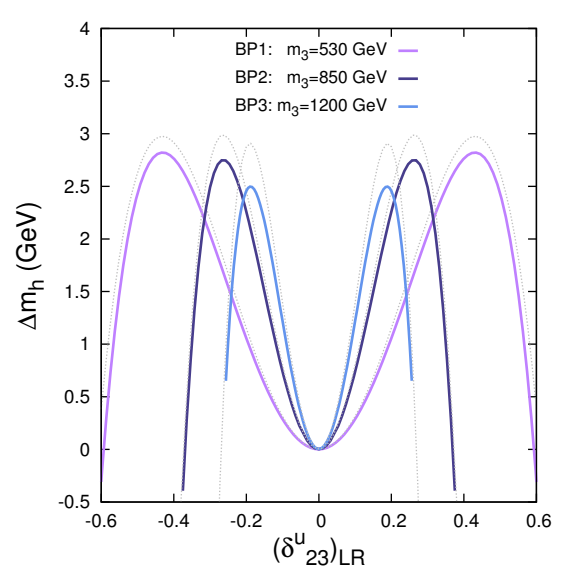

(a)

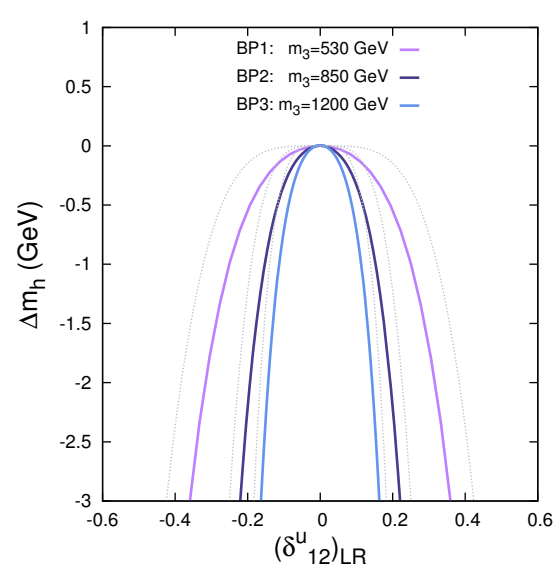

(b)

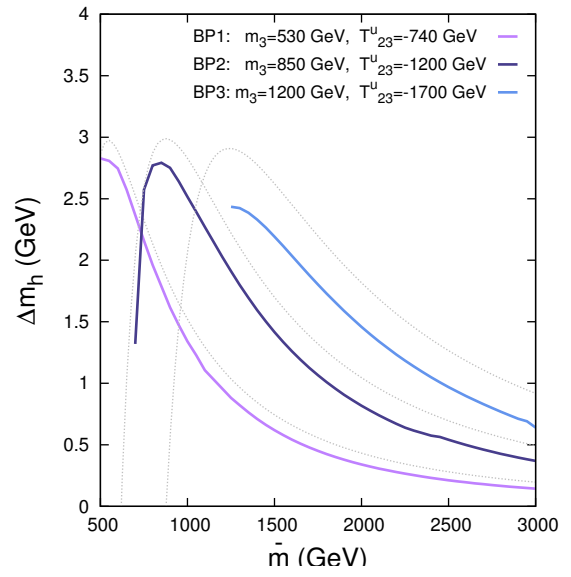

(c)

Figure 1. The enhancement of the Higgs boson mass $m_{h}$ due to flavour violating parameters (a) $\left(\delta_{23}^{u}\right)_{L R}$ and (b) $\left(\delta_{12}^{u}\right)_{L R}$ for the benchmark points defined in table 1. (c) Dependence of $\Delta m_{h}^{2}$ on the supersymmetric mass scale $\tilde{m}$ for fixed values of $T_{23}^{u}$. Thick solid lines indicate the results form SPheno while the thin ones show the predictions of analytical formulae (2.10) and (2.11).

Case 4: GFV and non-universal diagonal entries. An additional effect arises when a mass hierarchy between the diagonal entries of the matrix $\left(m_{\tilde{U}}^{2}\right)_{L R}$ is observed. Let us consider a kind of inverted hierarchy scenario, assuming that the first two generations of up squarks have the same common mass $\tilde{m}_{2}$, while the mass of the third generation is given by $\tilde{m}_{3}$. An approximate analytical formula in the case of the soft mass splitting in the stop sector have been derived in ref. [4]. It is straightforward to extend those results for the case of the inter-generation mixing, using as a reference eq. (2.10) . Assuming that only $\left(\delta_{23}^{u}\right)_{L R} \neq 0$ one obtains

$$
\begin{aligned}
\Delta m_{h}^{2}\left(\left(\delta_{23}^{u}\right)_{L R}\right)= & \frac{3}{4 \pi^{2}}\left\{( \delta _ { 2 3 } ^ { u } ) _ { L R } ^ { 2 } \frac { \tilde { m } _ { 2 } ^ { 2 } \tilde { m } _ { 3 } ^ { 2 } } { \tilde { m } _ { 2 } ^ { 2 } - \tilde { m } _ { 3 } ^ { 2 } } \left[\frac{1}{2} Y_{t}^{2} \sin ^{2} \beta \ln \left(\frac{\tilde{m}_{2}^{2}}{\tilde{m}_{3}^{2}}\right)\right.\right. \\
& \left.\left.+\left(\delta_{23}^{u}\right)_{L R}^{2} \frac{\tilde{m}_{2}^{2} \tilde{m}_{3}^{2}}{v^{2}\left(\tilde{m}_{2}^{2}-\tilde{m}_{3}^{2}\right)}\left(2-\frac{\tilde{m}_{2}^{2}+\tilde{m}_{3}^{2}}{\tilde{m}_{2}^{2}-\tilde{m}_{3}^{2}} \ln \left(\frac{\tilde{m}_{2}^{2}}{\tilde{m}_{3}^{2}}\right)\right)\right]\right\} .
\end{aligned}
$$




\begin{tabular}{|c|c|c|c|c|c|c|c|c|c|c|}
\hline & $M_{1}$ & $M_{2}$ & $M_{3}$ & $A_{t, b, \tau}$ & $\mu$ & $m_{A}$ & $\tan \beta$ & $m_{\tilde{L}, \tilde{e}}$ & $m_{\tilde{Q}_{1,2}, \tilde{u}_{1,2}, \tilde{d}}$ & $m_{\tilde{Q}_{3}, \tilde{u}_{3}}$ \\
\hline BP4 & 300 & 160 & 1600 & 0 & 236 & 1665 & 39 & 3000 & 5000 & 756 \\
BP5 & 300 & 160 & 1600 & 0 & 189 & 1310 & 49 & 3000 & 5000 & 842 \\
BP6 & 300 & 160 & 1600 & 0 & 236 & 1410 & 49 & 3000 & 5000 & 990 \\
\hline
\end{tabular}

Table 2. Input parameters for benchmark points BP4 - BP6 at $M_{\mathrm{SUSY}}$. The masses and trilinear terms are in $\mathrm{GeV}$.

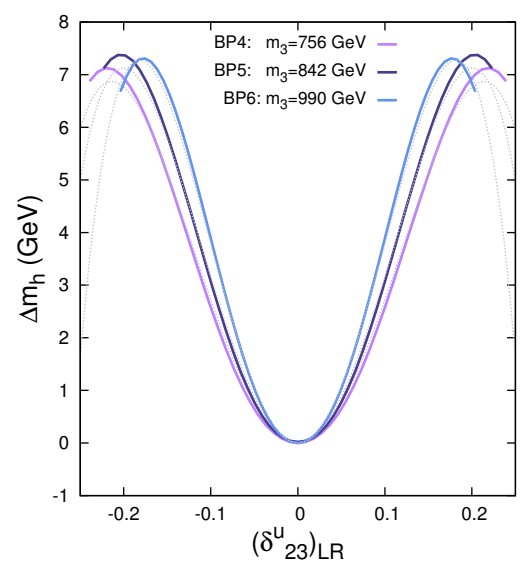

(a)

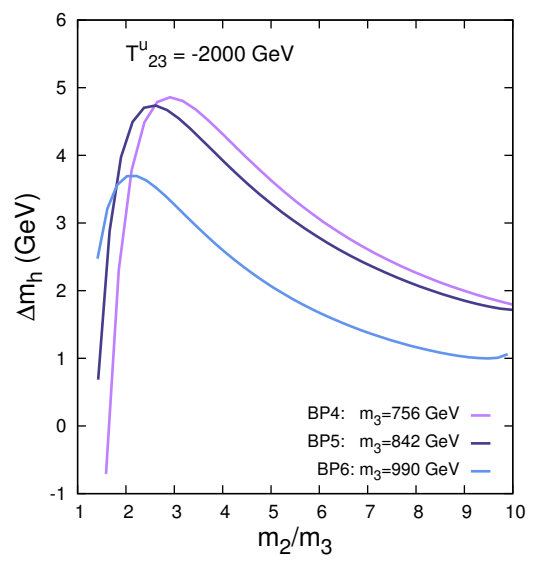

(b)

Figure 2. (a) The enhancement of the Higgs boson mass $m_{h}$ due to flavour violating parameter $\left(\delta_{23}^{u}\right)_{L R}$. Thick solid lines indicate the results form SPheno while the thin gray ones show the predictions of analytical formula (2.12). (b) Dependence of $\Delta m_{h}$ on the mass splitting $\tilde{m}_{2} / \tilde{m}_{3}$. The benchmark points are defined in table 2 .

An immediate consequence of eq. (2.12) is that the Higgs mass correction due to $\left(\delta_{23}^{u}\right)_{L R}$ can be strongly enhanced by the mass splitting between the first/second and the third generation.

In figure 2(a) we show the dependence of $m_{h}$ on $\left(\delta_{23}^{u}\right)_{L R}$ for three benchmark points defined in table 2. The soft masses of the first two generations were moved to $5 \mathrm{TeV}$ to account for the effects of large mass splitting, while the heaviness of right-handed sbottom further reduces the impact from the sbottom mixing, which is now of the order of $0.02 \mathrm{GeV}^{2}$. Once more the analytical results (thin grey lines) are compared with the output from SPheno (thick lines). As was expected, in this case the enhancement of the Higgs boson mass can be more than twice as large as in the universal mass case, reaching up to $7-8 \mathrm{GeV}$.

In figure 2(b) we show the dependence of $\Delta m_{h}$ on the mass difference between scharm and stop for our three benchmark points. In all the cases we fixed the value of the offdiagonal entry $\left(T_{u}\right)_{23}$ in the SCKM-basis, $\left(T_{u}\right)_{23}=-2000 \mathrm{GeV}$. One can observe that the effect is maximalised for the mass ratio $\tilde{m}_{2} / \tilde{m}_{3}$ at around $2-4$, while further splitting tends to reduce it. 


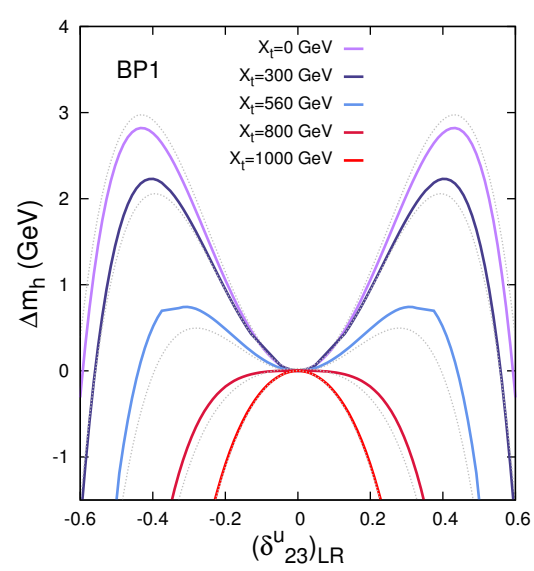

(a)

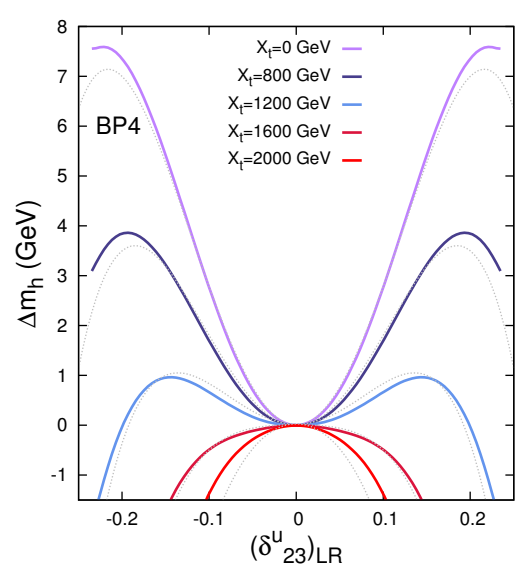

(b)

Figure 3. The enhancement of the Higgs boson mass $m_{h}$ due to flavour violating parameter $\left(\delta_{23}^{u}\right)_{L R}$ and stop mixing term $\tilde{X}_{t}$ for benchmark points (a) BP1, and (b) BP4. Thick solid lines indicate the results form SPheno while the thin ones show the predictions of analytical formulae (2.13) and (2.14).

Case 5: impact of $\tilde{X}_{t} \neq 0$. So far we have limited our discussion to the case with no mixing in the stop sector, $\tilde{X}_{t}=0$. In the presence of non-zero $\tilde{X}_{t}$ term, the Higgs mass correction takes the form

$$
\Delta m_{h}^{2}=\frac{3}{4 \pi^{2}} \tilde{m}^{2}\left[Y_{t}^{2} \sin ^{2} \beta\left(\left(\delta_{33}^{u}\right)^{2}+\frac{1}{2}\left(\delta_{23}^{u}\right)_{L R}^{2}\right)-\frac{\tilde{m}^{2}}{6 v^{2}}\left(\left(\delta_{33}^{u}\right)^{4}+\left(\delta_{23}^{u}\right)_{L R}^{4}+2\left(\delta_{33}^{u}\right)^{2}\left(\delta_{23}^{u}\right)_{L R}^{2}\right)\right]
$$

for the universal sfermion masses, and

$$
\begin{aligned}
\Delta m_{h}^{2}\left(\left(\delta_{23}^{u}\right)_{L R}\right)= & \frac{3}{4 \pi^{2}}\left\{Y_{t}^{2} \sin ^{2} \beta\left[\left(\delta_{33}^{u}\right)^{2} \tilde{m}_{3}^{2}+\frac{1}{2}\left(\delta_{23}^{u}\right)_{L R}^{2} \frac{\tilde{m}_{2}^{2} \tilde{m}_{3}^{2}}{\tilde{m}_{2}^{2}-\tilde{m}_{3}^{2}} \ln \left(\frac{\tilde{m}_{2}^{2}}{\tilde{m}_{3}^{2}}\right)\right]\right. \\
& +\left(\delta_{23}^{u}\right)_{L R}^{4} \frac{\tilde{m}_{2}^{4} \tilde{m}_{3}^{4}}{v^{2}\left(\tilde{m}_{2}^{2}-\tilde{m}_{3}^{2}\right)^{2}}\left(2-\frac{\tilde{m}_{2}^{2}+\tilde{m}_{3}^{2}}{\tilde{m}_{2}^{2}-\tilde{m}_{3}^{2}} \ln \left(\frac{\tilde{m}_{2}^{2}}{\tilde{m}_{3}^{2}}\right)\right)-\frac{\tilde{m}_{3}^{4}}{6 v^{2}}\left(\delta_{33}^{u}\right)^{4} \\
& \left.-\frac{\left(\delta_{33}^{u}\right)^{2}\left(\delta_{23}^{u}\right)_{L R}^{2}}{v^{2}}\left[\frac{2}{\sqrt{6}} \frac{\tilde{m}_{2}^{2} \tilde{m}_{3}^{4}}{\tilde{m}_{2}^{2}-\tilde{m}_{3}^{2}} \mid 2-\frac{\tilde{m}_{2}^{2}+\tilde{m}_{3}^{2}}{\tilde{m}_{2}^{2}-\tilde{m}_{3}^{2}} \ln \left(\frac{\tilde{m}_{2}^{2}}{\tilde{m}_{3}^{2}}\right)^{1 / 2}\right]\right\}(2 .)
\end{aligned}
$$

for the hierarchical case. We remind the reader that $\delta_{33}^{u}=\tilde{m} \frac{\tilde{X} v_{2}}{\sqrt{2}}$. The main qualitative difference with respect to the cases discussed above is the presence of a negative term $\sim\left(\delta_{33}^{u}\right)^{2}\left(\delta_{23}^{u}\right)_{L R}^{2}$. This new contribution can easily dilute the Higgs mass enhancement obatined through $\left(\delta_{23}^{u}\right)_{L R}$ if $\tilde{X}_{t}$ becomes large enough with respect to $\tilde{m}$ or $\tilde{m}_{3}$. We show this effect in figure 3 for benchmark points BP1 (panel (a)) and BP4 (panel (b)) and different choices of $\tilde{X}_{t}$ (corresponding to different colours of the solid lines). In particular one can observe that when the ratio $\tilde{X}_{t} / \tilde{m}$ becomes greater that $\sim 1.5$, it is no longer possible to increase $m_{h}$ through the non-zero parameter $\left(\delta_{23}^{u}\right)_{L R}$.

Case 6: off-diagonal entries in $\left(\boldsymbol{m}_{\tilde{U}}^{2}\right)_{L L}$ and $\left(\boldsymbol{m}_{\tilde{U}}^{2}\right)_{\boldsymbol{R} R}$. We will now evaluate the contribution to $m_{h}$ due to parameters $\left(\delta_{i j}^{u}\right)_{L L}$ and $\left(\delta_{i j}^{u}\right)_{R R}$. Note that in the GFV framework 
large off-diagonal elements $\left(\left(m_{\tilde{U}}^{2}\right)_{L L}\right)_{23} \sim \lambda^{2}\left(\tilde{m}_{2}^{2}-\tilde{m}_{3}^{2}\right)$, where $\lambda \sim 0.22$ is the Cabibbo angle, can naturally appear due to disalignement between the quark and squark fields if the mass difference between the diagonal elements of the matrix $\left(m_{Q}^{2}\right)_{L L}, \tilde{m}_{2}^{2}-\tilde{m}_{3}^{2}$, is large.

Let us consider for simplicity the universal case. The masses of the mixed left-handed eigenstates are given by $m_{1 L}^{2}=\frac{1}{2} m_{t}^{2}+\tilde{m}^{2}+\tilde{m}^{2}\left(\delta_{23}^{u}\right)_{L R}$ and $m_{2 L}^{2}=\frac{1}{2} m_{t}^{2}+\tilde{m}^{2}-\tilde{m}^{2}\left(\delta_{23}^{u}\right)_{L R}$ (assuming $\left(\delta_{23}^{u}\right)_{L L}>\frac{m_{t}^{2}}{2 \tilde{m}^{2}}$ ). Since $\left(\delta_{23}^{u}\right)_{L L}$ does not depend on $v_{2}$, it only results in a shift of the soft mass $\tilde{m}$ that lifts the degeneracy between previously universal soft masses of the third generation. In such a situation the Higgs mass is corrected by a factor [4]

$$
\Delta m_{h}^{2}\left(\left(\delta_{23}^{u}\right)_{L L}\right)=\frac{3}{8 \pi^{2}} \frac{m_{Z}^{4}}{v^{2}} \cos 2 \beta\left(1-\frac{8}{3} \sin ^{2} \theta_{W}\right)\left(\frac{m_{t}^{2}}{m_{Z}^{2}}+\frac{1}{6} \cos 2 \beta\right) \ln \left(1+\left(\delta_{23}^{u}\right)_{L L}\right) .
$$

This contribution is proportional to the mass scale of the EW sector and therefore negligible, unless the parameter $\left(\delta_{23}^{u}\right)_{L L}$ becomes very large. The same effect is observed for $\left(\delta_{13}^{u}\right)_{L L}$

On the other hand, the non-zero value of the parameter $\left(\delta_{12}^{u}\right)_{L L}$ does not influence $m_{h}$ at one-loop level, since it does not trigger the mixing with the third generation.

Case 7: more than one $\left(\delta_{i j / j i}^{u}\right)_{L R} \neq 0$. Finally, we will analyse the impact of two simultaneously non-zero parameters $\left(\delta_{i j / j i}^{u}\right)_{L R}$. The net effect strongly depends on which pairs are considered. If the GFV entries are present in two separate blocks of the matrix $\left(m_{\tilde{U}}^{2}\right)_{L R}$, namely upper and lower triangle, the individual contributions to $\Delta m_{h}$ will sum up allowing for much stronger enhancement of the Higgs boson mass. In figure 4(a) we show this effect for $\left(\delta_{23}^{u}\right)_{L R}=\left(\delta_{32}^{u}\right)_{L R}$ for three benchmark points defined in table 2 . It turns out that $m_{h}$ can be lifted up even by $14-15 \mathrm{GeV}$. The same effect would be observed in the case of $\left(\delta_{13}^{u}\right)_{L R}=\left(\delta_{31}^{u}\right)_{L R}$ and $\left(\delta_{23}^{u}\right)_{L R}=\left(\delta_{31}^{u}\right)_{L R}$. On the contrary, if one considers two GFV entries in the same block of $\left(m_{\tilde{U}}^{2}\right)_{L R}$, the net effect will be limited as the parameter $\left(\delta_{23}^{u}\right)_{L R}$ in eq. (2.10) and eq. (2.12) will be replaced by $\delta_{\text {eff }}=\sqrt{\left(\delta_{i j}^{u}\right)_{L R}^{2}+\left(\delta_{i k}^{u}\right)_{L R}^{2}}$. This can be seen in figure $4(\mathrm{~b})$ for $\left(\delta_{23}^{u}\right)_{L R}=\left(\delta_{13}^{u}\right)_{L R}$.

Note that in this case the diluting effect, observed for $\tilde{X}_{t}$, is absent as a negative mixed term $\sim\left(\delta_{23}^{u}\right)_{L R}\left(\delta_{32}^{u}\right)_{L R}$ does not appear.

\section{Limits on $\left(\delta_{13}^{u}\right)_{L R},\left(\delta_{31}^{u}\right)_{L R},\left(\delta_{23}^{u}\right)_{L R}$ and $\left(\delta_{32}^{u}\right)_{L R}$}

In this section we will discuss the consistency of the GFV scenarios analysed above with both the experimental data and a theoretical requirement of the vacuum stability. In the former case, the strongest limits on the allowed values of the off-diagonal entries in the SSB terms come from the measurements of the FCNC transitions. The influence of various flavour changing processes on the corresponding parameters $\delta_{i j}$ have been thoroughly discussed in the literature, see for example refs. [35, 36]. Interestingly, the four most important parameters from the point of view of the Higgs boson mass enhancement, namely $\left(\delta_{13}^{u}\right)_{L R},\left(\delta_{31}^{u}\right)_{L R},\left(\delta_{23}^{u}\right)_{L R}$, and $\left(\delta_{32}^{u}\right)_{L R}$, are very weakly bounded by flavour constraints. In fact, there are only a few ways of constraining the $(1,3)$ and $(2,3)$ sectors of the left-right mixing blocks in $\mathcal{M}_{\tilde{u}}^{2}$. 




(a)

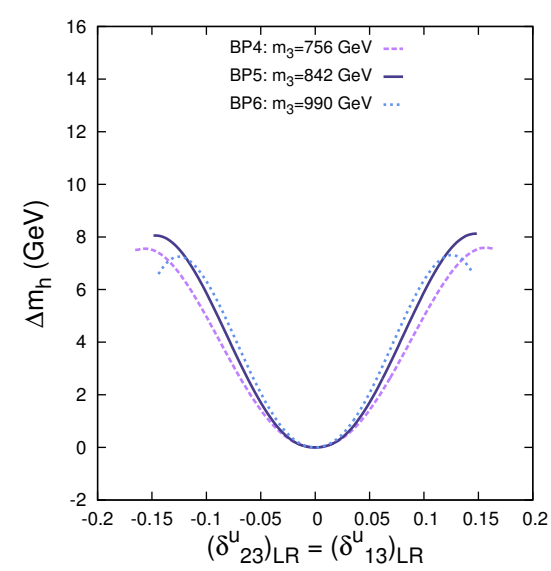

(b)

Figure 4. The enhancement of the Higgs boson mass $m_{h}$ due to flavour violating parameters (a) $\left(\delta_{23}^{u}\right)_{L R}=\left(\delta_{32}^{u}\right)_{L R}$, and $(\mathrm{b})\left(\delta_{23}^{u}\right)_{L R}=\left(\delta_{13}^{u}\right)_{L R}$. The benchmark points defined in table 2.

The chargino-squark loop contributions to rare decays $b \rightarrow s \gamma, b \rightarrow d \gamma, B_{s} \rightarrow \mu^{+} \mu^{-}$, as well as to $\bar{B}_{s}^{0}-B_{s}^{0}$ and $\bar{B}_{d}^{0}-B_{d}^{0}$ mixing, can provide upper bounds on the allowed values of the chirality flipping deltas. This possibility has been investigated in refs. [20] and [21], where the limits on $\left(\delta_{23}^{u}\right)_{L R}$ and $\left(\delta_{32}^{u}\right)_{L R}$ have been derived. The actual strength of the bounds clearly depends on the choice of the parameter space, though $\left(\delta_{32}^{u}\right)_{L R}$ as large as 0.22 can be excluded in certain cases, mainly through the measurement of $\mathrm{BR}\left(\overline{\mathrm{B}} \rightarrow \mathrm{X}_{\mathrm{s}} \gamma\right)$.

A chargino-loop contribution to the semileptonic decay $\bar{B} \rightarrow K^{*} l^{+} l^{-}$has been recently analysed in ref. [37]. It was shown that a strong bound $\left(\delta_{23}^{u}\right)_{L R}<0.1$ can be derived for $m_{\tilde{u}_{3}} \sim 300 \mathrm{GeV}$ and large stop mixing. A similar study has been performed in refs. [38, 39] for the $K \rightarrow \pi \nu \bar{\nu}$ decay and $\bar{K}^{0}-K^{0}$ mixing, providing bounds on $\left(\delta_{23}^{u}\right)_{L R}$ and $\left(\delta_{13}^{u}\right)_{L R}$. However, it should be emphasised that the actual limits depend on the flavour-diagonal MSSM masses and in general become effective only for relatively light up-squarks with masses below several hundred $\mathrm{GeV}$.

The other way of constraining the chiralilty changing entries of $\mathcal{M}_{\tilde{u}}^{2}$ would be through rare decays of the top quark, $t \rightarrow h c$ and $t \rightarrow h u$. A recent analysis by CMS, based on $19.5 / \mathrm{fb}$ of data at $\sqrt{s}=8 \mathrm{TeV}$, puts the upper limit on the $t \rightarrow h c$ branching ratio as $\operatorname{BR}(t \rightarrow h c)<5.6 \times 10^{-3}[40]$. This result, however, is still almost two orders of magnitude larger than the corresponding branching ratios calculated in the MSSM, $6 \times 10^{-5}[22,41,42]$, and henceforth does not provide constraints on the GFV parameters $\left(\delta_{23}^{u}\right)_{L R}$ and $\left(\delta_{32}^{u}\right)_{L R}$.

In table 3 we present the limits on the flavour-violating parameters $\left(\delta_{13}^{u}\right)_{L R},\left(\delta_{31}^{u}\right)_{L R}$, $\left(\delta_{23}^{u}\right)_{L R}$ and $\left(\delta_{32}^{u}\right)_{L R}$ for six benchmark points defined in table 1 and table 2, obtained by considering the following FCNC processes: $b \rightarrow s \gamma, B_{s} \rightarrow \mu^{+} \mu^{-}, B_{d} \rightarrow \mu^{+} \mu^{-}, \Delta M_{B_{s}}$, $K^{0} \rightarrow \pi^{0} \nu \bar{\nu}$ and $K^{+} \rightarrow \pi^{+} \nu \bar{\nu}$. The flavour observables were calculated with the public code SUSY_FLAVOR v2.10 [43], and the following experimental measurements were applied: $\mathrm{BR}\left(\overline{\mathrm{B}} \rightarrow \mathrm{X}_{\mathrm{s}} \gamma\right)=3.43 \pm 0.3 \times 10^{-4}[44], \mathrm{BR}\left(\mathrm{B}_{\mathrm{s}} \rightarrow \mu^{+} \mu^{-}\right)=2.9 \pm 0.76 \times 10^{-9}[45,46]$, $\mathrm{BR}\left(\mathrm{B}_{\mathrm{d}} \rightarrow \mu^{+} \mu^{-}\right)<7.4 \times 10^{-10}[45,46], \Delta M_{B_{s}}=1.166 \pm 0.158 \times 10^{-11} \mathrm{GeV}[24], \mathrm{BR}\left(K^{0} \rightarrow\right.$ 


\begin{tabular}{|c|c|c|c|c|c|c|c|}
\hline & BP1 & BP2 & BP3 & BP4 & BP5 & BP6 & FCNC process \\
\hline$\left(\delta_{23}^{u}\right)_{L R}$ & {$[-0.87: 0.55]$} & - & - & - & - & - & $b \rightarrow s \gamma$ \\
$\left(\delta_{32}^{u}\right)_{L R}$ & {$[-0.2: 0.52]$} & - & - & {$[-0.15: 0.3]$} & {$[-0.16: 0.19]$} & {$[-0.2:-]$} & $b \rightarrow s \gamma\left(B_{s} \rightarrow \mu^{+} \mu^{-}\right)$ \\
$\left(\delta_{13}^{u}\right)_{L R}$ & - & - & - & - & - & - & - \\
$\left(\delta_{31}^{u}\right)_{L R}$ & {$[-0.45: 0.55]$} & - & - & - & - & - & $K^{+} \rightarrow \pi^{+} \nu \bar{\nu}$ \\
\hline
\end{tabular}

Table 3. Limits on the parameters $\left(\delta_{13}^{u}\right)_{L R},\left(\delta_{31}^{u}\right)_{L R},\left(\delta_{23}^{u}\right)_{L R}$ and $\left(\delta_{32}^{u}\right)_{L R}$ from the FCNC processes discussed in the text. The benchmark points are defined in table 1 and table 2 . In the last column a process that provides the strongest bound is shown.

$\left.\pi^{0} \nu \bar{\nu}\right)<2.6 \times 10^{-8}[24], \mathrm{BR}\left(K^{+} \rightarrow \pi^{+} \nu \bar{\nu}\right)=1.73 \pm 1.15 \times 10^{-10}$ [24], where theoretical and experimental errors were added in quadrature. When deriving the limits on $\delta_{i j}$, a $2 \sigma$ deviation from the experimentally measured central value was allowed.

One can observe that the benchmark point BP1 is the only one that can be significantly constrained by the FCNC processes. This had to be expected as BP1 is characterised by the lightest SUSY spectrum. More importantly, however, even after imposing the FCNC constraints the maximal Higgs mass enhancement through all parameters $\delta_{i j}$ discussed in the previous section is still possibile. One can also notice that $b \rightarrow s \gamma$ seems to be the strongest experimental constraint, which is in agreement with the findings of refs. [20] and [21]. Interestingly, it can provide stringent bounds on the allowed values of parameter $\left(\delta_{32}^{u}\right)_{L R}$ for the points with the hierarchical up-squark matrices (mainly due to the large values of $\tan \beta$ ), which however remain consistent with the values required to increase $m_{h}$.

The off-diagonal entries in the SSB matrices can also be bounded by requiring that the radiative corrections to the CKM elements generated through the squark-gluino loops do not exceed the experimental values, as discussed in refs. [47-49]. In fact, such limits can be stronger than the FCNC ones if SUSY spectrum is heavier than $500 \mathrm{GeV}$ and they become even more effective when the squark mass increases. The bound on $\left(\delta_{31}^{u}\right)_{L R}$ is particularly important in that context as it was shown to be very strong [47-49].

In table 4 we present the limits on the flavour-violating parameters $\left(\delta_{13}^{u}\right)_{L R},\left(\delta_{31}^{u}\right)_{L R}$, $\left(\delta_{23}^{u}\right)_{L R}$, and $\left(\delta_{32}^{u}\right)_{L R}$ after the inclusion of chirally enhanced corrections to CKM matrix elements. The corrections were calculated with SUSY_FLAVOR v2.10 and the following experimental values were assumed: $V_{t s}=0.0429 \pm 0.0026, V_{c b}=0.0409 \pm 0.0011, V_{u b}=$ $0.00415 \pm 0.00049$ [24]. One can immediately see that the impact of those additional constraints is dramatic. The non-zero values of $\left(\delta_{31}^{u}\right)_{L R}$ are now totally excluded by demanding that a radiative correction to $V_{u b}$ does not exceed the experimentally measured value. Also $\left(\delta_{32}^{u}\right)_{L R}$ becomes very strongly constrained. However, the Higgs mass enhancement is still perfectly possible with $\left(\delta_{13}^{u}\right)_{L R} \neq 0$ as this parameter remains virtually unconstrained.

Additional bounds on the flavour-violating parameters $\left(\delta_{13}^{u}\right)_{L R},\left(\delta_{31}^{u}\right)_{L R},\left(\delta_{23}^{u}\right)_{L R}$ and $\left(\delta_{32}^{u}\right)_{L R}$ arise from the requirement that the $\mathrm{EW}$ vacuum is stable. The scalar potential of the MSSM can develop a charge/color breaking (CCB) minimum lower than the EW one, or it can become unbounded from below (UFB), if the trilinear couplings are too large [5054]. More importantly, unlike the FCNC constraints, these kind of bounds do not weaken when $M_{\text {SUSY }}$ increases. In the case of the up-squark sector the corresponding formulae for 


\begin{tabular}{|c|c|c|c|c|c|c|}
\hline & BP1 & BP2 & BP3 & BP4 & BP5 & BP6 \\
\hline$\left(\delta_{23}^{u}\right)_{L R}$ & {$[-0.45: 0.35]$} & {$[-0.15: 0.12]$} & {$[-0.14: 0.12]$} & excl. & excl. & excl. \\
$\left(\delta_{32}^{u}\right)_{L R}$ & {$[-0.05: 0.04]$} & {$[-0.02: 0.02]$} & {$[-0.05: 0.02]$} & {$[-0.04: 0.01]$} & {$[-0.04: 0.01]$} & {$[-0.02: 0.01]$} \\
$\left(\delta_{13}^{u}\right)_{L R}$ & - & - & - & - & - & - \\
$\left(\delta_{31}^{u}\right)_{L R}$ & excl. & excl. & excl. & excl. & excl. & excl. \\
\hline
\end{tabular}

Table 4. Limits on the parameters $\left(\delta_{13}^{u}\right)_{L R},\left(\delta_{31}^{u}\right)_{L R},\left(\delta_{23}^{u}\right)_{L R}$ and $\left(\delta_{32}^{u}\right)_{L R}$ from the FCNC processes discussed in the text and the chirally enhanced corrections to CKM matrix elements. The benchmark points are defined in table 1 and table 2 .

\begin{tabular}{|c|c|c|c|c|c|c|}
\hline & BP1 & BP2 & BP3 & BP4 & BP5 & BP6 \\
\hline CCB & $\leq 0.47$ & $\leq 0.28$ & $\leq 0.20$ & $\leq 0.23$ & $\leq 0.21$ & $\leq 0.18$ \\
UFB & $\leq 4.90$ & $\leq 0.52$ & $\leq 0.44$ & $\leq 0.30$ & $\leq 0.27$ & $\leq 0.23$ \\
\hline
\end{tabular}

Table 5. CCB and UFB vacuum stability bounds on the parameters $\left(\delta_{13}^{u}\right)_{L R},\left(\delta_{31}^{u}\right)_{L R},\left(\delta_{23}^{u}\right)_{L R}$ and $\left(\delta_{32}^{u}\right)_{L R}$. The benchmark points are defined in table 1 and table 2 .

the CCB limits are given by [54]

$$
\left(\delta_{i 3}^{u}\right)_{L R} \leq m_{t} \frac{\left[\left(m_{\tilde{U}}^{2}\right)_{L L}^{i i}+\left(m_{\tilde{U}}^{2}\right)_{R R}^{33}+m_{H_{u}}^{2}+\mu^{2}\right]^{1 / 2}}{\left(m_{\tilde{U}}\right)_{R R}^{i i}\left(m_{\tilde{U}}\right)_{L L}^{33}}, \quad i=1,2,
$$

and the bounds on $\left(\delta_{3 i}^{u}\right)_{L R}$ are obtained by switching the indices 3 and $i$. Similarly, the UFB limits read [54]

$$
\left(\delta_{i 3}^{u}\right)_{L R} \leq m_{t} \frac{\left[\left(m_{\tilde{U}}^{2}\right)_{L L}^{i i}+\left(m_{\tilde{U}}^{2}\right)_{R R}^{33}+\left(m_{\tilde{L}}^{2}\right)_{L L}^{i i}+\left(m_{\tilde{L}}^{2}\right)_{R R}^{33}\right]^{1 / 2}}{\left(m_{\tilde{U}}\right)_{R R}^{i i}\left(m_{\tilde{U}}\right)_{L L}^{33}}, \quad i=1,2 .
$$

Note that the denominator in the above expressions differ from the one shown in [54] due to different normalization of the parameters $\delta_{i j}$ in eq. (2.7).

In table 5 we present the vacuum stability bounds on the flavour-violating parameters $\left(\delta_{13}^{u}\right)_{L R},\left(\delta_{31}^{u}\right)_{L R},\left(\delta_{23}^{u}\right)_{L R}$, and $\left(\delta_{32}^{u}\right)_{L R}$ for our six benchmark points. The limits are the same for all considered deltas due to the fact that two first generations of sfermions are degenerate. The CCB bounds are much more constraining than the UFB ones, mainly due to the presence of heavy sleptons in the spectrum. This effect is particularly strong for the benchmark points BP1-BP3, characterized by relatively light squarks. One can also observe that the bounds become stronger when the masses of the up-squark increase. It should be emphasised, however, that the derived limts are consistent with the value of parameters $\delta_{i j}$ necessary for the maximal or nearly maximal enhacement of the Higgs boson mass.

On the other hand, the appearance of the CCB minimum does not necessarily need to be a problem for a model, as long as the lifetime of the EW vacuum is longer than the age of the universe. The derivation of the metastability limits is quite complex and involves numerical calculation of the bounce action for a given scalar potential. Such an analysis was performed in ref. [56] in the context of the off-diagonal trilinear terms. It was shown that the resulting metastability bounds do not depend on the Yukawa couplings and 
therefore are in general much less stringent than the CCB ones. The effect is, however, least significant for $\left(\delta_{13}^{u}\right)_{L R},\left(\delta_{31}^{u}\right)_{L R},\left(\delta_{23}^{u}\right)_{L R}$, and $\left(\delta_{32}^{u}\right)_{L R}$, where the upper limit from vacuum stability requirement can be weakened by only $10-15 \%$.

\section{GFV effects in GUT-constrained scenarios}

In the previous section we showed that the mass of the lightest Higgs boson can be significantly enhanced by non-zero chirality changing mixing between the third and second/first generations, triggered by the presence of non-zero off-diagonal entries in the trilinear coupling matrix $\left(m_{\tilde{U}}^{2}\right)_{L R}$. In this section we will analyse the corresponding GFV effects in the framework of GUT-constrained scenarios. Here the dependence of $m_{h}$ on the parameters $\delta_{i j}$ is slightly more complicated, as one must take into account the RGE evolution of the input parameters between the scales $M_{\mathrm{GUT}}$ and $M_{\mathrm{SUSY}}$.

While working in the framework of General Flavour Violation, it is convenient to rewrite the corresponding RGEs in the SCKM-basis. Then the input parameters at the GUT-scale are defined in the basis in which the Yukawa matrices are diagonal and the flavour violating effects manifest themselves through the presence of the CKM-matrix elements in the RG equations. From now on we will always assume that the soft terms at $M_{\mathrm{GUT}}$ are given in the SCKM-basis.

Let us first analyse the impact of the non-zero element $\left(T_{u}\right)_{23}$ in the up-squark trilinear matrix $T_{u}$. The only RGEs directly affected by its presence (neglecting the contribution from $g_{1}$ and $g_{2}$ and all the Yukawa couplings but $Y_{t}$ ) are:

$$
\begin{aligned}
\frac{d\left(T_{u}\right)_{23}}{d t} & =\frac{1}{16 \pi^{2}}\left(T_{u}\right)_{23}\left(8 Y_{t}^{2}-\frac{16}{3} g_{3}^{2}\right), \\
\frac{m_{H_{u}}^{2}}{d t} & =\frac{6}{16 \pi^{2}}\left(Y_{t}^{2} m_{H_{u}}^{2}+Y_{t}^{2} m_{\tilde{Q}_{3}}^{2}+Y_{t}^{2} m_{\tilde{u}_{3}}^{2}+\sum_{i}\left(T_{u}\right)_{i i}^{2}+\left(T_{u}\right)_{23}^{2}\right),
\end{aligned}
$$

where we used the formulae given in [57]. Therefore, two effects are present while evolving the soft SUSY-breaking parameters down from the GUT scale: a) reduction of the soft squark masses due to a positive contribution to $m_{H_{u}}$ from $\left(T_{u}\right)_{23}$ and, b) increase of the term $\left(T_{u}\right)_{23}$ driven by top-Yukawa. Both factors make $\left(\delta_{23}^{u}\right)_{L R}$ at $M_{\text {SUSY }}$ increase, thus facilitating the $m_{h}$ enhancement. On the other hand, lower squark masses mean lowering of the Higgs boson mass with respect to the MFV case. Therefore, in order to increase the scalar mass $m_{h}$ in a GUT-constrained scenario, the former effect should dominate the latter.

In figure $5(\mathrm{a})$ we show the dependence $\Delta m_{h}$ vs $\left(\delta_{23}^{u}\right)_{L R}$ for five benchmark points with mSUGRA inspired universal boundary conditions at the GUT scale. The input values of the soft parameters are given in table 6 (labelled with $\mathrm{C}$ ) and the off-diagonal entry $\left(T_{u}\right)_{23}\left(M_{\mathrm{GUT}}\right)$, denoted as $T_{0}$, is varied in the range [-3000:3000] $\mathrm{GeV}$. The reference value of the Higgs boson mass in the case of Minimal Flavour Violation corresponds to $T_{0}=0$. In figure $5(\mathrm{~b})$ we present the same mass dependence as a function of $T_{0}$. As one can see, the enhancement of the Higgs boson mass with respect to the MVF case is still possible, but its magnitude is mitigated by the RG running so it does not exceed 


\begin{tabular}{|c|c|c|c|c|c|c|c|c|c|c|}
\hline & $\mathrm{C} 1$ & $\mathrm{C} 2$ & $\mathrm{C} 3$ & $\mathrm{C} 4$ & $\mathrm{C} 5$ & $\mathrm{I} 1$ & $\mathrm{I} 2$ & $\mathrm{I} 3$ & $\mathrm{I} 4$ & $\mathrm{I} 5$ \\
\hline$m_{0}(1,2)$ & 2320 & 1320 & 1320 & 900 & 900 & 8000 & 5000 & 5000 & 3000 & 3000 \\
$m_{1 / 2}$ & 1380 & 1080 & 1080 & 508 & 508 & 1380 & 1080 & 1080 & 508 & 508 \\
$A_{0}$ & 0 & 0 & 2000 & 0 & 1000 & 0 & 0 & 1000 & 0 & 1000 \\
$\tan \beta$ & 31 & 28 & 28 & 18 & 18 & 31 & 28 & 28 & 18 & 18 \\
\hline$m_{0}(3)$ & 2320 & 1320 & 1320 & 900 & 900 & 2320 & 1320 & 1320 & 900 & 900 \\
\hline
\end{tabular}

Table 6. Input parameters for benchmark points at $M_{\mathrm{GUT}}$. Letter $\mathrm{C}$ refers to the CMSSM boundary conditions while I to the inverted hierarchy scenario. The masses and trilinear terms are in $\mathrm{GeV}$.

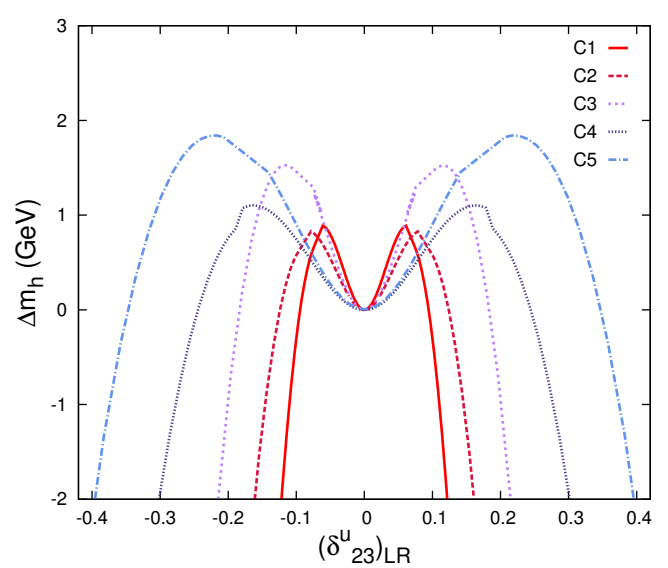

(a)

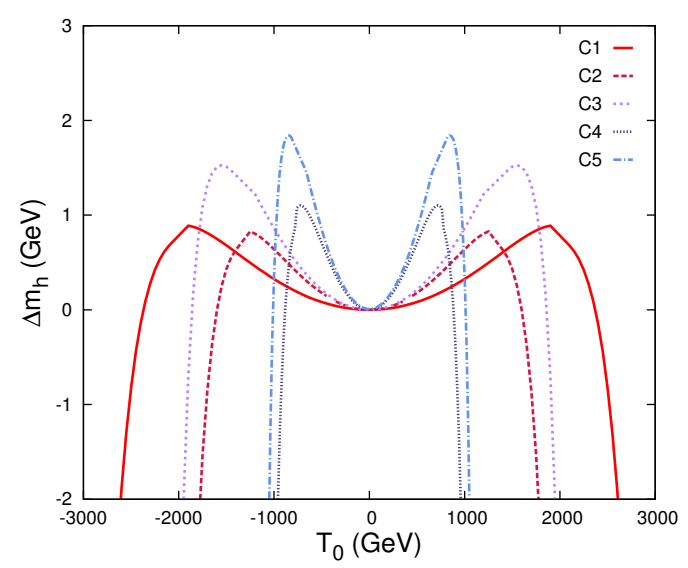

(b)

Figure 5. The enhancement of the Higgs boson mass $m_{h}$ due to parameter $T_{0}$ as a function of (a) $\left(\delta_{23}^{u}\right)_{L R}$, and (b) $T_{0}$ in the CMSSM. The benchmark points are defined in table 6 .

$2 \mathrm{GeV}$. The effect is distinctly strongest for the benchmark points $\mathrm{C} 3$ and $\mathrm{C} 5$, which are characterised by the ratio $A_{0} / m_{1 / 2} \simeq 2$.

To better understand the mechanism underlying this behaviour, let us write down approximate formulae that quantify the dependence of the relevant soft SUSY-breaking terms at the EW scale on the input GUT-scale parameters:

$$
\begin{aligned}
\left(m_{\tilde{Q}}^{2}\right)_{33}\left(M_{\mathrm{EW}}\right) & \simeq 2.96 m_{1 / 2}^{2}-0.07 A_{0}^{2}-0.31 T_{0}^{2}+0.14 A_{0} m_{1 / 2}+0.73 m_{0}^{2}, \\
\left(m_{\tilde{U}}^{2}\right)_{22}\left(M_{\mathrm{EW}}\right) & \simeq 3.56 m_{1 / 2}^{2}+0.1 A_{0}^{2}-0.96 T_{0}^{2}-0.03 A_{0} m_{1 / 2}+0.97 m_{0}^{2}, \\
\left(T_{u}\right)_{23}\left(M_{\mathrm{EW}}\right) & \simeq-0.04 m_{1 / 2}+1.34 T_{0} .
\end{aligned}
$$

The expansion coefficients multiplying the input parameters in the above polynomial have been calculated for the benchmark point C3 with SPheno_v.3.2.4. Since they only depend on the RGE running of the masses and trilinear terms, they do not change over the parameter space by more than $10-20 \%$ and the qualitative conclusions derived for a sample point are quite universal. From eq. (4.2) it is easy to see that with all other 


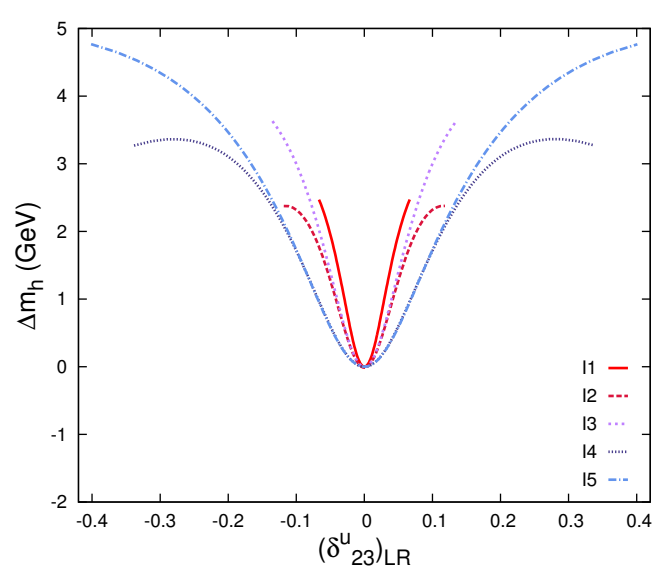

(a)



(b)

Figure 6. The enhancement of the Higgs boson mass $m_{h}$ due to parameter $T_{0}$ as a function of (a) $\left(\delta_{23}^{u}\right)_{L R}$, and (b) $T_{0}$ in the inverted hierarchy scenario. The benchmark points are defined in table 6 .

parameters fixed, larger $m_{0}$ allows for larger values of $T_{0}$ before any of the soft mass $\left(m_{\tilde{Q}}^{2}\right)_{33}$ or $\left(m_{\tilde{U}}^{2}\right)_{22}$ becomes negative at $M_{\mathrm{EW}}$. That is confirmed by figure $5(\mathrm{~b})$ while comparing the maximal allowed $T_{0}$ for the points $\mathrm{C} 1, \mathrm{C} 3$ and $\mathrm{C} 5$. Of course, for the points with larger $m_{0}$ the maximal $T_{0}$ corresponds to smaller $\left(\delta_{23}^{u}\right)_{L R}$, as the suppression of its value due to soft masses is stronger than the enhancement through $T_{0}$. Therefore in figure 5 (a) the pattern of benchmark points is inverted.

The impact of $A_{0}$ is somehow more subtle. With all other parameters fixed, larger $A_{0}$ also makes it easier to obtain larger $T_{0}$. This effect is, however, much weaker than the one due to $m_{0}$, since $\left(m_{\tilde{U}}^{2}\right)_{22}$ essentially does not depend on $A_{0}$ and is quickly driven below zero when $T_{0}$ increases. This behaviour is particularly clear when both $m_{0}$ and $m_{1 / 2}$ are small, as can be seen for the points $\mathrm{C} 4$ and $\mathrm{C} 5$ for which the maximum $T_{0}$ is almost the same. On the other hand, exactly the same feature makes it possible to enhance the Higgs mass more than in the case with $A_{0}=0$. A closer look at eq. (4.2) shows that for a given set of input parameters there exists a range of $T_{0}$ when even a small change in its value results in a quick drop of $\left(m_{\tilde{U}}^{2}\right)_{22}\left(M_{\mathrm{EW}}\right)$ and, subsequently, a quick rise of $\left(\delta_{23}^{u}\right)_{L R}$ (this tends to happen for smaller $T_{0}$ when $m_{0}$ becomes smaller). Therefore, larger $A_{0}$ allows for slightly larger $T_{0}$ and that is enough to enhance $m_{h}$.

On the other hand, it was pointed out in section 2 that the effects due to intergeneration mixing can be stronger in the case of large mass splitting between stops and scharms of different chiralities. In figure $6\left(\right.$ a) we show the dependence $\Delta m_{h}$ vs $\left(\delta_{23}^{u}\right)_{L R}$ for five benchmark points defined in table 6 (labelled with I), and in figure 6(b) the dependence $\Delta m_{h}$ vs $T_{0}$. All the input parameters but $m_{0}(1,2)$ are equal to the ones analysed in the case with universal boundary conditions. The amount of mass splitting between the first/second and third generations have been chosen to maximalise the impact of $\left(\delta_{23}^{u}\right)_{L R}$, as discussed in section 2. One can see that in this scenario the Higgs boson mass can be enhanced 


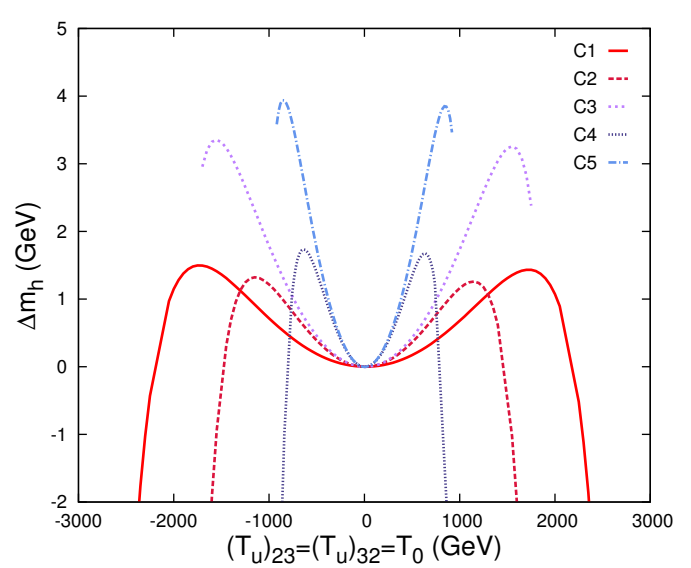

(a)

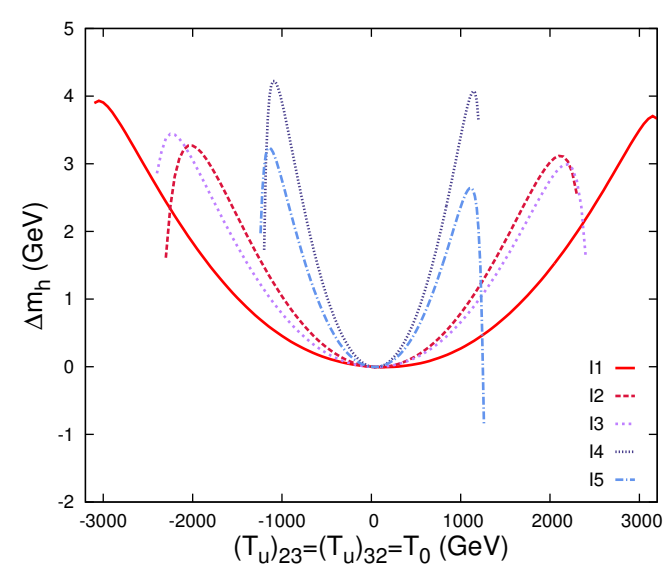

(b)

Figure 7. The enhancement of the Higgs boson mass $m_{h}$ due to parameter $\left(T_{u}\right)_{32}=\left(T_{u}\right)_{23}=T_{0}$ for (a) CMSSM, and (b) inverted hierarchy scenario. The benchmark points are defined in table 6 .

more than in the CMSSM, up to $5 \mathrm{GeV}$. This is a direct consequence of the mass splitting between the up-squarks. The EW-scale value of $\left(m_{\tilde{U}}^{2}\right)_{22}$ is now modified as

$$
\left(m_{\tilde{U}}^{2}\right)_{22}\left(M_{\mathrm{EW}}\right) \simeq 4.6 m_{1 / 2}^{2}+1.16 A_{0}^{2}-1.05 T_{0}^{2}+0.01 A_{0} m_{1 / 2}-0.03 m_{0}^{2}(3)+0.24 m_{0}^{2}(1,2),
$$

so its dependence on $T_{0}$ is weaker with respect to the dependence on other parameters. As a result, the drop of the soft masses due to $T_{0}$ is now much slower and $\left(\delta_{23}^{u}\right)_{L R}$ can be largely increased before $\left(m_{\tilde{Q}}^{2}\right)_{33}$ becomes negative.

Finally we will analyse the case with both $\left(T_{u}\right)_{23}$ and $\left(T_{u}\right)_{32}$ different from zero and equal to $T_{0}$ at the GUT-scale. After the discussion in section 2 one could expect that the enhancement of the Higgs boson mass will be now much stronger. Figure 7 shows that while it is in fact possible for the CMSSM (panel (a)), it is totally not the case in the inverted hierarchy scenario (panel (b)). The reason is once more the RGE running. For the IH mass pattern the renormalization of the soft term $\left(m_{\tilde{Q}}^{2}\right)_{33}$ due to both off-diagonal entries is so strong that it quickly dominates the effect of the increased $T_{0}$.

To summarise the findings of this section, we will briefly comment on possible implications of the GFV contributions to the Higgs boson mass on the phenomenology of two scenarios discussed above, and in particular on the allowed masses of stops and gluinos (the full phenomenological analysis is a subject of the next section). In figure 8 we show the iso-contours of $m_{h}$ in the $\left(m_{\tilde{t}_{1}}, m_{\tilde{g}}\right)$ plain for (a) CMSSM, and (b) inverted hierarchy scenario with $m_{0}(1,2)=3 m_{0}(3)$. The universal scalar and gaugino masses where scanned freely, while the remaining parameters were kept fixed at $A_{0}=0$ and $\tan \beta=30$. The colours correspond to different values of the off-diagonal entry $\left(T_{u}\right)_{23}=T_{0}$ at the GUT scale: $T_{0}=0$ (gray), $T_{0}=-2000 \mathrm{GeV}$ (dark red), $T_{0}=-3000 \mathrm{GeV}$ (light blue). The lines break when reaching the region of the parameter space corresponding either to no EWSB, or to tachyonic stop, or to the sequence of stop and gluino masses that cannot be obtained 


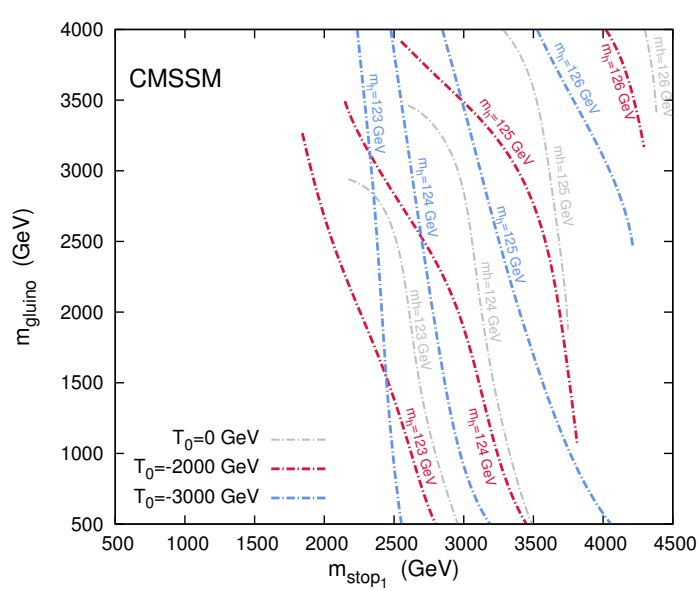

(a)



(b)

Figure 8. Iso-contours of $m_{h}$ in the $\left(m_{\tilde{t}_{1}}, m_{\tilde{g}}\right)$ plain for (a) CMSSM, and (b) inverted hierarchy with $m_{0}(1,2)=3 m_{0}(3)$. In both plots $A_{0}=0, \tan \beta=30$, while the off-diagonal entry $\left(T_{u}\right)_{23}=T_{0}$ is set at $T_{0}=0$ (gray), $T_{0}=-2000 \mathrm{GeV}$ (dark red), and $T_{0}=-3000 \mathrm{GeV}$ (light blue).

given the input parameter range. We do not show those regions explicitly on the plots as their position depends on the choice of $T_{0}$.

From figure 8 one can see that the impact of $T_{0}$ is particularly important in the inverted hierarchy case. If $T_{0}=-3000 \mathrm{GeV}$, the Higgs boson with the mass at $125 \mathrm{GeV}$ can be obtained for $m_{\tilde{t}_{1}}=1370 \mathrm{GeV}, m_{\tilde{g}}=2500 \mathrm{GeV}$ and a small stop-sector mixing $\left|X_{t} / M_{\mathrm{SUSY}}\right|=0.82$. On the other hand, if $T_{0}=0$ the corresponding value of $m_{h}$ requires $m_{\tilde{t}_{1}}=2850 \mathrm{GeV}$ and $m_{\tilde{g}}=3600 \mathrm{GeV}$.

On the contrary, the impact of non-zero $T_{0}$ in the case of the CMSSM is not particularly strong.

\section{Global analysis}

So far we have discussed the possibility of enhancing the Higgs boson mass through the GFV corrections to the scalar potential without questioning the validity of such scenarios when confronted with the experimental data. To address this issue, in this section we will study the phenomenology of the GUT-constrained inverted hierarchy scenario in the context of its predictions about the relic density of dark matter, EW precision observables and flavour physics.

The numerical analysis was performed with the package BayesFITSv3.1, described in detail in [5]. The package is linked to MultiNest v2.7 [59] for sampling. Mass spectra are calculated with SPheno v3.2.4; the branching ratios $\mathrm{BR}\left(\overline{\mathrm{B}} \rightarrow \mathrm{X}_{\mathrm{s}} \gamma\right), \mathrm{BR}\left(\mathrm{B}_{\mathrm{s}} \rightarrow \mu^{+} \mu^{-}\right)$ and $\mathrm{BR}\left(\mathrm{B}_{\mathrm{u}} \rightarrow \tau \nu\right)$ as well as mass difference $\Delta M_{B_{s}}$ and SUSY contribution to anomalous magnetic moment of the muon $\delta(g-2)_{\mu}^{\mathrm{SUSY}}$ with SUSY_FLAVOR v2.10; the relic density and spin-independent neutralino-proton cross section $\sigma_{p}^{\mathrm{SI}}$ with DarkSUSY v5.0.6 [60]; and EW precision constraints with FeynHiggs v2.10.0. To include the exclusion limits from 


\begin{tabular}{|l|l|l|l|}
\hline Measurement & Mean or range & Error: exp., th. & Ref. \\
\hline$m_{h}($ by CMS $)$ & $125.7 \mathrm{GeV}$ & $0.4 \mathrm{GeV}, 3 \mathrm{GeV}$ & {$[3]$} \\
$\Omega_{\chi} h^{2}$ & 0.1199 & $0.0027,10 \%$ & {$[23]$} \\
$\mathrm{BR}\left(\overline{\mathrm{B}} \rightarrow \mathrm{X}_{\mathrm{s}} \gamma\right) \times 10^{4}$ & 3.43 & $0.22,0.21$ & {$[44]$} \\
$\mathrm{BR}\left(\mathrm{B}_{\mathrm{s}} \rightarrow \mu^{+} \mu^{-}\right) \times 10^{9}$ & 2.9 & $0.7,10 \%$ & {$[45,46]$} \\
$\mathrm{BR}\left(\mathrm{B}_{\mathrm{u}} \rightarrow \tau \nu\right) \times 10^{4}$ & 0.72 & $0.27,0.38$ & {$[58]$} \\
$\Delta M_{B_{s}} \times 10^{11}$ & $1.166 \mathrm{GeV}$ & $0.008 \mathrm{GeV}, 0.158 \mathrm{GeV}$ & {$[24]$} \\
$\sin ^{2} \theta_{\mathrm{eff}}$ & 0.23146 & $0.00012,0.00015$ & {$[24]$} \\
$M_{W}$ & $80.385 \mathrm{GeV}$ & $0.015 \mathrm{GeV}, 0.015 \mathrm{GeV}$ & {$[24]$} \\
\hline$M_{t}$ & $173.34 \mathrm{GeV}$ & $0.76 \mathrm{GeV}, 0$ & {$[24]$} \\
$m_{b}\left(m_{b}\right)^{\overline{M S}}$ & $4.18 \mathrm{GeV}$ & $0.03 \mathrm{GeV}, 0$ & {$[24]$} \\
$\alpha_{\mathrm{em}}^{-1}$ & 127.916 & $1.00 .015,0$ & {$[24]$} \\
$\alpha_{s}$ & 0.1184 & $0.0007,0$ & {$[24]$} \\
\hline
\end{tabular}

Table 7. The experimental constraints applied in the analysis.

Higgs boson searches at LEP, Tevatron, and the LHC and the $\chi^{2}$ contributions from the Higgs boson signal rates from Tevatron and the LHC we use HiggsBounds v1.0.0 [61-63] interfaced with Higgsignals v1.0.0 [64]. The SM parameters $\left(M_{t}, m_{b}\left(m_{b}\right)^{\overline{M S}}, \alpha_{\mathrm{em}}\right.$ and $\left.\alpha_{s}\right)$ where treated as nuisance parameters and randomly drawn from a Gaussian distribution around the central value. The experimental constraints applied in the analysis are listed in table 7 . Note that we do not assign any statistical interpretation to the presented results.

We scanned the input parameters of the model in the following ranges:

$$
\begin{aligned}
100 \mathrm{GeV} & \leq m_{0}(3) \leq 6000 \mathrm{GeV} & & 1 \leq m_{0}(1,2) / m_{0}(3) \leq 10, \quad 100 \mathrm{GeV} \leq m_{1 / 2} \leq 3000 \mathrm{GeV} \\
3 & \leq \tan \beta \leq 62, & & -3000 \mathrm{GeV} \leq T_{0} \leq-1000 \mathrm{GeV} .
\end{aligned}
$$

The universal trilinear coupling was set as $A_{0}=0$. This choice comes from the fact that our main interest is to investigate the possible enhancement of $m_{h}$ in the situation when the mixing in the stop sector is far from its maximal value. For the same reason we scanned the parameter $T_{0}$ far from zero.

In figure 9 we show the distribution of the model points in the planes corresponding to the input parameters: (a) $\left(m_{0}(3), m_{1 / 2}\right)$, (b) $\left(m_{0}(1,2), m_{0}(3)\right)$, (c) $\left(\tan \beta, T_{0}\right)$. The points presented as grey squares satisfy $2 \sigma$ bounds on the Higgs mass and the relic density, while the ones depicted as green circles additionally belong to $2 \sigma$ acceptance region for the $\mathrm{EW}$ precision observables $m_{W}$ and $\sin ^{2} \theta_{\text {eff }}$. Finally, the brown stars correspond to those points that satisfy at $2 \sigma$ all the experimental constraints listed in table 7 .

The requirement of satisfying the bound on the relic density is usually the most stringent one. The only dark matter candidate in the analysed scenario is the lightest neutralino. In the narrow region spanned around $m_{0} \approx 1 \mathrm{TeV}$ it is predominantly bino-like and the proper value of the relic density is obtained through neutralino co-annihilation with the 


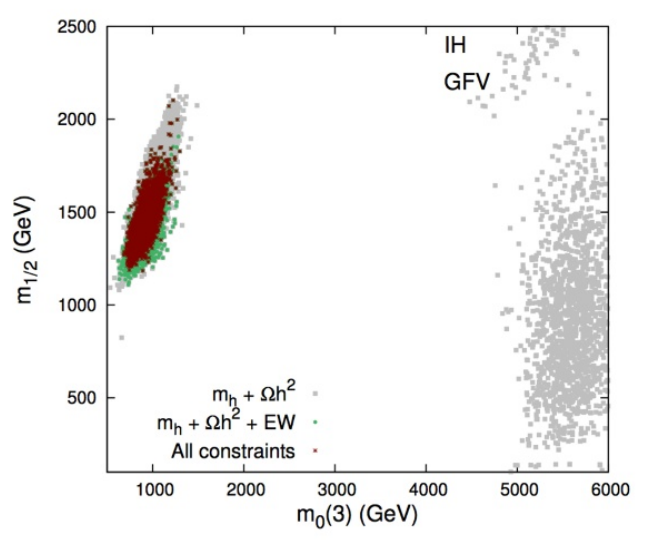

(a)

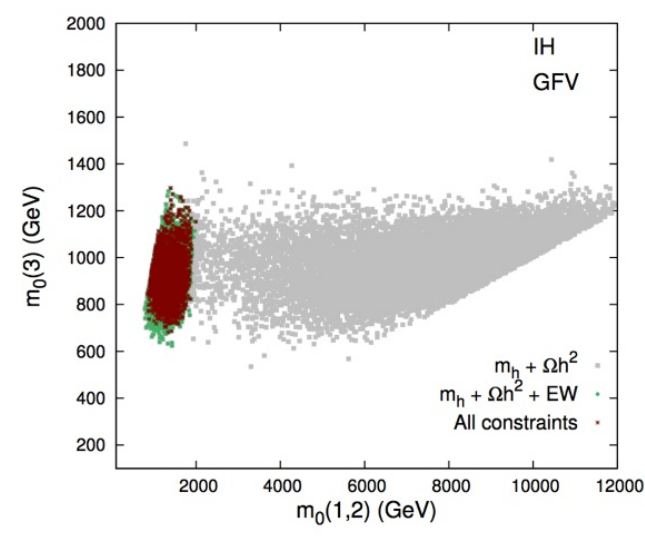

(b)



(c)

Figure 9. Scatter plots of the IH scenario points in the planes of (a) $\left(m_{0}(3), m_{1 / 2}\right)$, (b) $\left(m_{0}(1,2)\right.$, $\left.m_{0}(3)\right)$, and (c) $\left(\tan \beta, T_{0}\right)$. The grey squares correspond to the points satisfying the $2 \sigma$ bounds on the Higgs mass and the relic density, the green circles additionally satisfy the $2 \sigma$ bound on the EW precision observables, while the brown crosses all the constraints listed in table 7 .

lightest slepton (stau). The corresponding value of $\tan \beta$ is limited to $40-60$, while the off-diagonal entry $T_{0}$ remains unconstrained. On the other hand, in a vast region with $m_{0}$ between $5-6 \mathrm{TeV}$ neutralino is a mixture of bino and higgsinos. Subsequently, the annihilation cross-section is enhanced by a non-zero higgsino component that opens a possibility of efficient annihilation into gauge bosons through a t-channel exchange of higgsino-like $\tilde{\chi}_{1}^{ \pm}$ and $\tilde{\chi}_{2}^{0}$. In figure $9(\mathrm{c})$ this region corresponds to a narrow strip around $T_{0}=-1200 \mathrm{GeV}$ spanned over the wide range of $\tan \beta$, and it is not shown in figure 9(b).

The situation severely changes after imposing constraints from the EW precision measurements. The whole part of the parameter space corresponding to $m_{0}(3)>1500 \mathrm{GeV}$ and $m_{0}(1,2)>2000 \mathrm{GeV}$ is now disfavoured at $2 \sigma$ level. This is a direct consequence of abandoning the MFV assumption. After the matrix $\left(m_{\tilde{Q}}^{2}\right)_{i j}$ is rotated to the SCKM-basis, an off-diagonal element $\left(\left(m_{\tilde{U}}^{2}\right)_{L L}\right)_{23} \sim \lambda^{2}\left(\tilde{m}_{2}^{2}-\tilde{m}_{3}^{2}\right)$ is generated. Since in the inverted hierarchy scenario the corresponding mass difference between the diagonal entries is large, the 


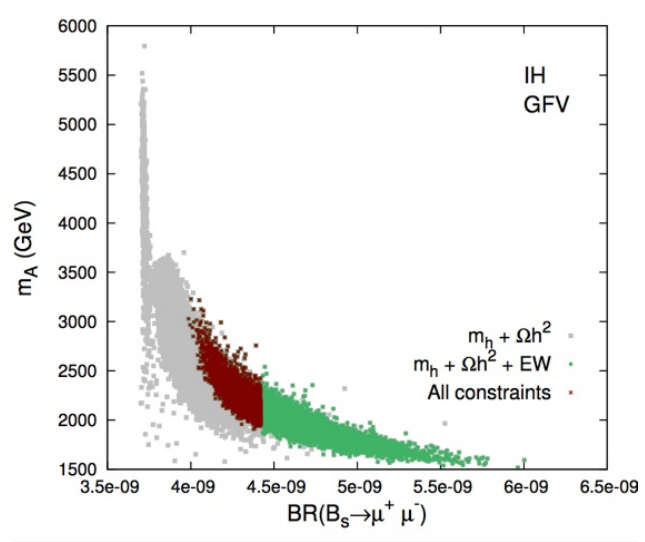

(a)



(c)

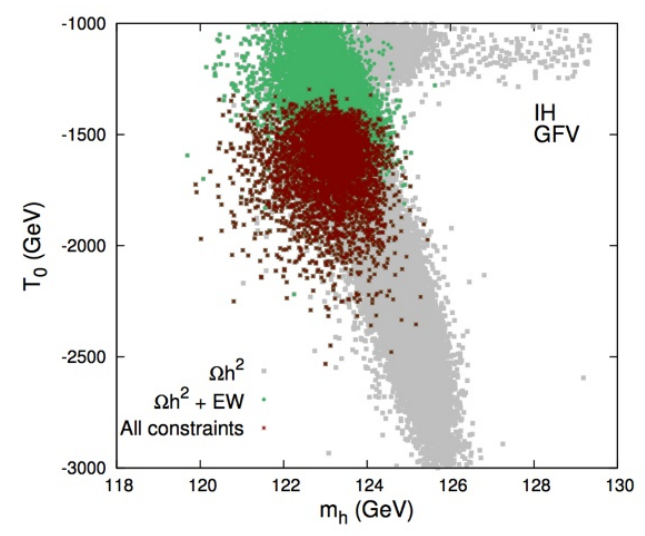

(b)

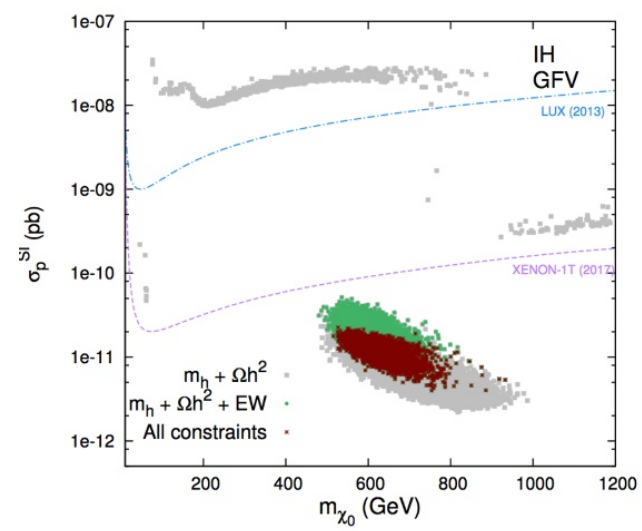

(d)

Figure 10. Scatter plots of the IH scenario points in the planes of (a) $\left(\mathrm{BR}\left(\mathrm{B}_{\mathrm{s}} \rightarrow \mu^{+} \mu^{-}\right), m_{A}\right),(\mathrm{b})$ $\left(T_{0}, m_{h}\right),(\mathrm{c})\left(m_{\tilde{t}_{1}}, m_{\tilde{g}}\right),(\mathrm{d})\left(m_{\tilde{\chi}_{1}^{0}}, \sigma_{p}^{\mathrm{SI}}\right)$. The colour code is the same as in figure 9 .

flavour violating term $\left(\left(m_{\tilde{U}}^{2}\right)_{L L}\right)_{23}$ is substantial and induces large 1-loop corrections to the electroweak parameter $\rho$, and consequently to $m_{W}$ and $\sin ^{2} \theta_{\text {eff }}$, as discussed in details in ref. [18]. One can also observe an upper bound on the gaugino mass term $m_{1 / 2}<1800 \mathrm{GeV}$ in the $\tilde{\tau}$-coannihilation region that translates into a lower bound on $T_{0}>-2500 \mathrm{GeV}$. It results from the fact that as long as $m_{0}(3)$ remains low, the squark soft masses become much more strongly renormalised when $m_{1 / 2}$ increases. In effect, $M_{\mathrm{SUSY}}$ also increases and the impact of the off-diagonal entry $\left(\left(m_{\tilde{U}}^{2}\right)_{L L}\right)_{23}$ is stronger.

Finally, after all the experimental constraints listed in table 7 are taken into account, the only parameter that becomes further affected is $T_{0}$, now disfavoured above $-1400 \mathrm{GeV}$. The main reason here is a tension with $\mathrm{BR}\left(\mathrm{B}_{\mathrm{s}} \rightarrow \mu^{+} \mu^{-}\right)$measurement. A supersymmetric contribution to this branching ratio is driven by a factor $\mathrm{BR}\left(\mathrm{B}_{\mathrm{s}} \rightarrow \mu^{+} \mu^{-}\right) \sim \frac{\tan \beta^{6}}{\mathrm{~m}_{\mathrm{A}}^{4}}[65]$. In figure 10(a) we show a distribution of the analysed points in the $\left(\operatorname{BR}\left(B_{s} \rightarrow \mu^{+} \mu^{-}\right), m_{A}\right)$ plane. Comparing this plot with figure $9(\mathrm{c})$ one can observe that in the $\tilde{\tau}$-coannihilation region mass of the pseudoscalar $A$ significantly decreases when $\left|T_{0}\right|$ becomes smaller, while 
in the mixed bino-higgsino region it stays always above $2500 \mathrm{GeV}$. Additionally, in the former case $\tan \beta$ is bound to be large. Remembering that $m_{A}^{2} \sim-m_{H_{u}}^{2}$ for large $\tan \beta$, this behaviour can be qualitatively understood from an approximate relation between the value of $m_{H_{u}}^{2}$ at the EW-scale and the GUT-scale input parameters:

$$
\begin{aligned}
\left(m_{H_{u}}^{2}\right)\left(M_{\mathrm{EW}}\right) \simeq & -0.96 m_{1 / 2}^{2}-0.40 A_{0}^{2}-0.38 T_{0}^{2}+0.61 A_{0} m_{1 / 2} \\
& +0.02 m_{0}^{2}(3)+0.003 m_{0}^{2}(1,2),
\end{aligned}
$$

which was derived for a sample point $m_{1 / 2}=1500 \mathrm{GeV}, m_{0}(3)=1000 \mathrm{GeV}, m_{0}(1,2)=$ $1000 \mathrm{GeV}, T_{0}=-1500 \mathrm{GeV}, \tan \beta=50$ and $A_{0}=0$. It is now clear from eq. (5.2) that the mass of a CP-odd scalar is enhanced when the value of GFV entry $T_{0}$ increases, making it easier to accommodate predictions of the model within the experimental bounds. In particular, we checked in a supplementary scan that when $T_{0}=0$, the whole parameter space of the analysed scenario is disfavoured by the $\mathrm{BR}\left(\mathrm{B}_{\mathrm{s}} \rightarrow \mu^{+} \mu^{-}\right)$constraint.

We would like to emphasise the importance of this effect. When the MFV assumption is abandoned, the experimental measurement of the branching ratio for rare decay $B_{s} \rightarrow \mu^{+} \mu^{-}$strongly disfavours inverted hierarchy scenarios unless large $T_{0}$ is introduced. Interestingly, the same parameter can be responsible for enhancement of the Higgs boson mass without the need of introducing large stop mixing.

We conclude this section with a brief discussion of the most relevant phenomenological features characterising the considered model. In figure 10 we present distributions of points in the planes of (a) $\left(\mathrm{BR}\left(\mathrm{B}_{\mathrm{s}} \rightarrow \mu^{+} \mu^{-}\right), m_{A}\right)$, (b) $\left(T_{0}, m_{h}\right)$, (c) $\left(m_{\tilde{t}_{1}}, m_{\tilde{g}}\right)$, and (d) $\left(m_{\tilde{\chi}_{1}^{0}}, \sigma_{p}^{\mathrm{SI}}\right)$. The colour code is the same as in figure 9. From figure 10(b) one can see that for $m_{0}(3)$ fixed at $\sim 1000 \mathrm{GeV}$ the Higgs mass in the ballpark of $126 \mathrm{GeV}$ can be easily obtained by increasing $T_{0}$, as discussed in the previous sections. However, the bounds from the EW precision measurements strongly disfavour the region of $m_{h}>126 \mathrm{GeV}$.

The allowed spectrum of SUSY particles is characterised by the lightest up-squarks heavier than $1600 \mathrm{GeV}$ and gluinos heavier than $2500 \mathrm{GeV}$. While far beyond the reach of the LHC $8 \mathrm{TeV}$, spectra of that kind might be still accessible by the LHC $14 \mathrm{TeV}$ with $3000 / \mathrm{fb}$ of collected data. Bino-like neutralino can be as light as $500 \mathrm{GeV}$ in this scenario, therefore multi-jet signatures with large amount of missing energy seem to be a promising tool for testing such spectra in the proton colliders. On the other hand, they will probably remain beyond the reach of direct dark matter detection experiments, as the corresponding spin-independent proton-neutralino cross-section is in general too low to be tested. This can be inferred from figure 10(d) where the dashed lines show the $90 \%$ C.L. exclusion bound by LUX [66] (blue line) and a projected sensitivity by XENON1T [67] (purple line).

\section{Conclusions}

In this study we analysed the phenomenology of SUSY scenarios beyond the Minimal Flavour Violation. As the general topic is very broad, we concentrated on the effects from the up-squark sector only.

Firstly, we investigated the possibility of enhancing the Higgs boson mass through non-zero entries in the SSB squark matrices that generate additional contributions to the 
one-loop scalar potential. The largest effect was achieved through non-zero $(2,3),(3,2)$, $(1,3)$ and $(3,1)$ entries of the up-squark trilinear coupling in the absence of mixing in the stop sector. Interestingly, the GFV parameters responsible for the $m_{h}$ enhancement are not very strongly constrained neither by the FCNC transitions nor by the vacuum stability bounds, and therefore can be kept relatively large. We also showed that the effect can be stronger in a class of models where the first/second generation of the up squarks is heavier than the third one.

Secondly, we analysed the phenomenology of a class of GUT-constrained models that assume inverted mass hierarchy in the squark sector. Such scenarios are particularly sensitive to GFV effects as non-degenerate squarks of the second and third generations gives rise to a large flavour violating entry $\left(\left(m_{\tilde{U}}^{2}\right)_{L L}\right)_{23}$ after the rotation to the SCKM-basis. Although it does not affect the value of the Higgs boson mass, the presence of such an entry has other important consequences. First of all, it induces large loop contributions to the EW precision observables $m_{W}$ and $\sin ^{2} \theta_{\text {eff }}$. Consequently, the allowed parameter space of the model is strongly limited to the values of $m_{0}(3)$ below $1500 \mathrm{GeV}$. Secondly, the same entry strongly reduces the mass of the CP-odd Higgs boson in the $\tilde{\tau}$-coannihilation strip, leading to dangerously large enhancement of $\mathrm{BR}\left(\mathrm{B}_{\mathrm{s}} \rightarrow \mu^{+} \mu^{-}\right)$. This effect, however, can be counterbalanced by the presence of a large non-zero $(2,3)$ entry in the SSB trilinear matrix of the up squarks.

The latter observation suggests that once the MFV assumption is lifted, a no-trivial GFV structure in the up squark sector is required for all the experimental constraints to be satisfied. Moreover, it was shown in ref. [68] that mixing between the right-handed charm and top squarks can reduce the experimental LHC bound on the stop mass, explaining the hitherto null result of the SUSY direct searches.

This might be a hint that supersymmetry, if realised in nature, should be considered in a more general, flavour violating framework.

\section{Acknowledgments}

I would like to thank Werner Porod for claryfing some issues related to usage of SPheno in the GFV framework. I am also very grateful to Enrico Maria Sessolo for many helpful discussions and comments on this manuscript. This work has been supported by the EU and MSHE Grant No. POIG.02.03.00-00-013/09. The use of the CIS computer cluster at the National Centre for Nuclear Research is gratefully acknowledged.

\section{A Derivation of the formulae for $\Delta m_{h}$}

In this appendix we present the details of our derivation of the analytical formulae for the GFV contributions to the lightest Higgs scalar mass shown in section 2. The approach is based on section 6 of ref. [32].

We start with defining a one-loop corrected scalar potential of the MSSM as

$$
V_{1}=V_{0}+\Delta V_{1}
$$


where $V_{0}$ is the tree-level potential and the one-loop radiative contribution in the dimensional reduction $(\overline{D R})$ scheme is given by a well know Coleman-Weinberg formula [69]

$$
\Delta V_{1}=\frac{1}{64 \pi^{2}} \operatorname{Str}\left[\mathcal{M}^{4}\left(\ln \frac{\mathcal{M}^{2}}{Q^{2}}-\frac{3}{2}\right)\right]
$$

where $Q$ denotes the renormalization scale, the supertrace is defined as $\operatorname{Str} F(\mathcal{M})=\sum_{i} C_{i}(-1)^{2 s_{i}}\left(2 s_{i}+1\right) F\left(m_{i}^{2}\right)$, and the sum goes over all the states in the theory. For a particle $i, C_{i}$ denotes the colour degrees of freedom and $s_{i}$ the spin. The mass matrix of the CP-even neutral Higgs bosons is given by the second derivatives of the one-loop corrected effective potential in its minimum,

$$
\mathcal{M}_{h^{0}, H^{0}}^{2}=\left.\frac{1}{2}\left(\begin{array}{cc}
\frac{\partial^{2} V_{1}}{\partial H_{2} \partial H_{2}^{*}} & \frac{\partial^{2} V_{1}}{\partial H_{2} \partial H_{1}^{*}} \\
\frac{\partial^{2} V_{1}}{\partial H_{1} \partial H_{2}^{*}} & \frac{\partial^{2} V_{1}}{\partial H_{1} \partial H_{1}^{*}}
\end{array}\right)\right|_{\mathrm{vev}} .
$$

In the decouplig limit, $m_{A} \gg m_{Z}$ where $A$ is the CP-odd scalar, the mass of the lightest Higgs boson is given by:

$$
m_{h}^{2}=\left(\mathcal{M}_{h^{0}, H^{0}}^{2}\right)_{11} \cos ^{2} \beta+\left(\mathcal{M}_{h^{0}, H^{0}}^{2}\right)_{22} \sin ^{2} \beta+\left(\mathcal{M}_{h^{0}, H^{0}}^{2}\right)_{12} \sin 2 \beta .
$$

Let us only consider the contribution to the scalar potential from the Higgs field and the up-squark sector. In such a simple case the only mass matrix entering eq. (A.2) is $\mathcal{M}_{\tilde{u}}^{2}$ defined by eq. (2.8),

$$
\Delta V_{1}=\frac{3}{64 \pi^{2}} \operatorname{tr}\left[\mathcal{M}_{\tilde{u}}^{4}\left(\ln \frac{\mathcal{M}_{\tilde{u}}^{2}}{Q^{2}}-\frac{3}{2}\right)-2 m_{t}^{4}\left(\ln \frac{m_{t}^{2}}{Q^{2}}-\frac{3}{2}\right)\right] .
$$

It is now convenient to decompose the matrix $\mathcal{M}_{\tilde{u}}^{2}$ as $\mathcal{M}_{\tilde{u}}^{2}=\tilde{m}^{2} \mathbb{I}+\mathcal{M}_{T}^{2}+\mathcal{M}_{F}^{2}$ (where the first term refers to the SSB mass contribution, $\mathcal{M}_{T}^{2}$ to the trilinear term contribution and $\mathcal{M}_{F}^{2}$ to the F-term superpotential contribution, respectively) and expand eq. (A.5) up to the fourth power of $\mathrm{H}_{2}$. Keeping only the quartic terms one gets:

$$
\Delta V_{1}=\frac{3}{64 \pi^{2}}\left[\ln \frac{\tilde{m}^{2}}{Q^{2}} \operatorname{tr}\left(\mathcal{M}_{F}^{2}\right)^{2}+\frac{1}{\tilde{m}^{2}} \operatorname{tr}\left(\left(\mathcal{M}_{T}^{2}\right)^{2} \mathcal{M}_{F}^{2}\right)-\frac{1}{12 \tilde{m}^{4}} \operatorname{tr}\left(\mathcal{M}_{T}^{2}\right)^{4}-2 m_{t}^{4}\left(\ln \frac{m_{t}^{2}}{Q^{2}}-\frac{3}{2}\right)\right] .
$$

Since $\mathcal{M}_{F}^{2}=m_{t}^{2} \mathbb{I}_{2}$, the first and the fourth term of eq. (A.6) combine to reproduce the oneloop leading logarithm term (where we used the minimalization condition $\partial \Delta V_{1} / \partial H_{2}=0$ ):

$$
\Delta\left(m_{h}^{2}\right)_{L L}=\frac{3}{8 \pi^{2} v^{2}} Y_{t}^{4} v_{2}^{4} \ln \frac{\tilde{m}^{2}}{m_{t}^{2}} .
$$

The remaining terms define the non-logarithmic finite corrections to the scalar potential which in general depends on the full structure of the mass matrix (2.8):

$$
\left(\Delta V_{1}\right)_{\text {finite }}=\frac{3}{64 \pi^{2}} \frac{1}{\tilde{m}^{2}}\left[\operatorname{tr}\left(\left(\mathcal{M}_{T}^{2}\right)^{2} \mathcal{M}_{F}^{2}\right)-\frac{1}{12 \tilde{m}^{2}} \operatorname{tr}\left(\mathcal{M}_{T}^{2}\right)^{4}\right] .
$$

As a first example let us consider the case when $\left(\delta_{23}^{u}\right)_{L R} \neq 0$. The terms in eq. (A.8) are then equal $\operatorname{tr}\left(\mathcal{M}_{T}^{2}\right)^{4}=2\left(T_{u}\right)_{23}^{4} H_{2}^{4}$ and $\operatorname{tr}\left(\left(\mathcal{M}_{T}^{2}\right)^{2} \mathcal{M}_{F}^{2}\right)=\left(T_{u}\right)_{23}^{2} H_{2}^{4}\left(Y_{t}^{2}+Y_{c}^{2}\right)$. Using 
eq. (A.3) and eq. (A.4) and neglecting the charm Yukawa coupling the GFV contribution to the Higgs mass takes the form

$$
\begin{aligned}
\Delta m_{h}^{2}\left(\left(\delta_{23}^{u}\right)_{L R}\right) & =\frac{3 v_{2}^{4}}{8 \pi^{2} v^{2}}\left[\frac{\left(T_{u}\right)_{23}^{2}}{\tilde{m}^{2}}\left(\frac{1}{2} Y_{t}^{2}-\frac{\left(T_{u}\right)_{23}^{2}}{12 \tilde{m}^{2}}\right)\right] \\
& =\frac{3}{4 \pi^{2}} \sin ^{2} \beta\left[\tilde{m}^{2}\left(\delta_{23}^{u}\right)_{L R}^{2}\left(\frac{1}{2} Y_{t}^{2}-\frac{\tilde{m}^{2}\left(\delta_{23}^{u}\right)_{L R}^{2}}{6 v_{2}^{2}}\right)\right] .
\end{aligned}
$$

As a second example let us evaluate the impact of non-zero stop mixing parameter $\tilde{X}_{t}=\left(T_{u}\right)_{33}$. In such a case the non-zero eigenvalues of the matrix $\left(\mathcal{M}_{T}^{2}\right)^{4}$ are again degenerate and read $\lambda_{1,2}=\left(\left(T_{u}\right)_{23}^{2}+\left(T_{u}\right)_{33}^{2}\right)^{2} H_{2}^{4}$. On the other hand, for the matrix $\left(\mathcal{M}_{T}^{2}\right)^{2} \mathcal{M}_{F}^{2}$ one obtains $\lambda_{1}=\left(\left(T_{u}\right)_{23}^{2}+\left(T_{u}\right)_{33}^{2}\right) H_{2}^{4} Y_{t}^{2}, \lambda_{2}=\left(T_{u}\right)_{33}^{2} H_{2}^{4} Y_{t}^{2}$. Therefore the traces give $\operatorname{tr}\left(\mathcal{M}_{T}^{2}\right)^{4}=2\left(\left(T_{u}\right)_{23}^{2}+\left(T_{u}\right)_{33}^{2}\right)^{2} H_{2}^{4}$ and $\operatorname{tr}\left(\left(\mathcal{M}_{T}^{2}\right)^{2} \mathcal{M}_{F}^{2}\right)=\left(2\left(T_{u}\right)_{33}^{2}+\left(T_{u}\right)_{23}^{2}\right) H_{2}^{4} Y_{t}^{2}$, and the one-loop correction to the Higgs boson mass takes the form

$$
\begin{aligned}
\Delta m_{h}^{2} & =\frac{3 v_{2}^{4}}{8 \pi^{2} v^{2} \tilde{m}^{2}}\left[Y_{t}^{2}\left(\left(T_{u}\right)_{33}^{2}+\frac{1}{2}\left(T_{u}\right)_{23}^{2}\right)-\frac{1}{12 \tilde{m}^{2}}\left(\left(T_{u}\right)_{33}^{4}+\left(T_{u}\right)_{23}^{4}+2\left(T_{u}\right)_{33}^{2}\left(T_{u}\right)_{23}^{2}\right)\right](\mathrm{A} .10) \\
& =\frac{3}{4 \pi^{2}} \tilde{m}^{2}\left[Y_{t}^{2} \sin ^{2} \beta\left(\left(\delta_{33}^{u}\right)^{2}+\frac{1}{2}\left(\delta_{23}^{u}\right)_{L R}^{2}\right)-\frac{\tilde{m}^{2}}{6 v^{2}}\left(\left(\delta_{33}^{u}\right)^{4}+\left(\delta_{23}^{u}\right)_{L R}^{4}+2\left(\delta_{33}^{u}\right)^{2}\left(\delta_{23}^{u}\right)_{L R}^{2}\right)\right] .
\end{aligned}
$$

Open Access. This article is distributed under the terms of the Creative Commons Attribution License (CC-BY 4.0), which permits any use, distribution and reproduction in any medium, provided the original author(s) and source are credited.

\section{References}

[1] CMS collaboration, Observation of a new boson at a mass of $125 \mathrm{GeV}$ with the CMS experiment at the LHC, Phys. Lett. B 716 (2012) 30 [arXiv:1207.7235] [INSPIRE].

[2] ATLAS collaboration, Observation of a new particle in the search for the Standard Model Higgs boson with the ATLAS detector at the LHC, Phys. Lett. B 716 (2012) 1 [arXiv: 1207.7214] [INSPIRE].

[3] CMS collaboration, Combination of Standard Model Higgs boson searches and measurements of the properties of the new boson with a mass near $125 \mathrm{GeV}$, CMS-PAS-HIG-13-005, CERN, Geneva Switzerland (2013).

[4] H.E. Haber, R. Hempfling and A.H. Hoang, Approximating the radiatively corrected Higgs mass in the minimal supersymmetric model, Z. Phys. C 75 (1997) 539 [hep-ph/9609331] [INSPIRE].

[5] A. Fowlie et al., The CMSSM favoring new territories: the impact of new LHC limits and a 125 GeV Higgs, Phys. Rev. D 86 (2012) 075010 [arXiv: 1206.0264] [INSPIRE].

[6] S. Akula, P. Nath and G. Peim, Implications of the Higgs boson discovery for mSUGRA, Phys. Lett. B 717 (2012) 188 [arXiv:1207.1839] [InSPIRE].

[7] C. Beskidt, W. de Boer, D.I. Kazakov and F. Ratnikov, Constraints on supersymmetry from LHC data on SUSY searches and Higgs bosons combined with cosmology and direct dark matter searches, Eur. Phys. J. C 72 (2012) 2166 [arXiv: 1207.3185] [INSPIRE]. 
[8] O. Buchmueller et al., The CMSSM and NUHM1 in light of $7 \mathrm{TeV} L H C, B_{s} \rightarrow \mu^{+} \mu^{-}$and XENON100 data, Eur. Phys. J. C 72 (2012) 2243 [arXiv:1207.7315] [InSPIRE].

[9] W. Altmannshofer, M. Carena, N.R. Shah and F. Yu, Indirect probes of the MSSM after the Higgs discovery, JHEP 01 (2013) 160 [arXiv:1211.1976] [INSPIRE].

[10] C. Strege et al., Global fits of the CMSSM and NUHM including the LHC Higgs discovery and new XENON100 constraints, JCAP 04 (2013) 013 [arXiv:1212.2636] [INSPIRE].

[11] M.E. Cabrera, J.A. Casas and R.R. de Austri, The health of SUSY after the Higgs discovery and the XENON100 data, JHEP 07 (2013) 182 [arXiv:1212.4821] [INSPIRE].

[12] K. Kowalska, L. Roszkowski and E.M. Sessolo, Two ultimate tests of constrained supersymmetry, JHEP 06 (2013) 078 [arXiv: 1302.5956] [INSPIRE].

[13] M. Badziak, E. Dudas, M. Olechowski and S. Pokorski, Inverted sfermion mass hierarchy and the Higgs boson mass in the MSSM, JHEP 07 (2012) 155 [arXiv:1205.1675] [INSPIRE].

[14] A. Arbey, M. Battaglia, A. Djouadi and F. Mahmoudi, The Higgs sector of the phenomenological MSSM in the light of the Higgs boson discovery, JHEP 09 (2012) 107 [arXiv: 1207.1348] [INSPIRE].

[15] J.R. Ellis, K. Enqvist, D.V. Nanopoulos and F. Zwirner, Observables in low-energy superstring models, Mod. Phys. Lett. A 1 (1986) 57 [INSPIRE].

[16] R. Barbieri and G.F. Giudice, Upper bounds on supersymmetric particle masses, Nucl. Phys. B 306 (1988) 63 [INSPIRE].

[17] A.G. Cohen, D.B. Kaplan and A.E. Nelson, The more minimal supersymmetric Standard Model, Phys. Lett. B 388 (1996) 588 [hep-ph/9607394] [INSPIRE].

[18] S. Heinemeyer, W. Hollik, F. Merz and S. Penaranda, Electroweak precision observables in the MSSM with nonminimal flavor violation, Eur. Phys. J. C 37 (2004) 481 [hep-ph/0403228] [INSPIRE].

[19] B. Herrmann, M. Klasen and Q. Le Boulc'h, Impact of squark flavour violation on neutralino dark matter, Phys. Rev. D 84 (2011) 095007 [arXiv: 1106.6229] [INSPIRE].

[20] M. Arana-Catania, S. Heinemeyer, M.J. Herrero and S. Penaranda, Higgs boson masses and B-physics constraints in non-minimal flavor violating SUSY scenarios, JHEP 05 (2012) 015 [arXiv:1109.6232] [INSPIRE].

[21] M. Arana-Catania, S. Heinemeyer and M.J. Herrero, Updated constraints on general squark flavor mixing, arXiv:1405.6960 [INSPIRE].

[22] J. Cao, G. Eilam, K.-I. Hikasa and J.M. Yang, Experimental constraints on stop-scharm flavor mixing and implications in top-quark FCNC processes, Phys. Rev. D 74 (2006) 031701 [hep-ph/0604163] [INSPIRE].

[23] Planck collaboration, P.A.R. Ade et al., Planck 2013 results. XVI. Cosmological parameters, Astron. Astrophys. (2014) [arXiv:1303.5076] [INSPIRE].

[24] Particle Data Group collaboration, J. Beringer et al., Review of particle physics (RPP), Phys. Rev. D 86 (2012) 010001 [INSPIRE].

[25] B.C. Allanach et al., SUSY Les Houches Accord 2, Comput. Phys. Commun. 180 (2009) 8 [arXiv:0801.0045] [INSPIRE]. 
[26] S. Heinemeyer, W. Hollik and G. Weiglein, The masses of the neutral CP-even Higgs bosons in the MSSM: accurate analysis at the two loop level, Eur. Phys. J. C 9 (1999) 343 [hep-ph/9812472] [INSPIRE].

[27] S. Heinemeyer, W. Hollik and G. Weiglein, FeynHiggs: a program for the calculation of the masses of the neutral CP-even Higgs bosons in the MSSM, Comput. Phys. Commun. 124 (2000) 76 [hep-ph/9812320] [INSPIRE].

[28] G. Degrassi, S. Heinemeyer, W. Hollik, P. Slavich and G. Weiglein, Towards high precision predictions for the MSSM Higgs sector, Eur. Phys. J. C 28 (2003) 133 [hep-ph/0212020] [INSPIRE].

[29] M. Frank et al., The Higgs boson masses and mixings of the complex MSSM in the Feynman-diagrammatic approach, JHEP 02 (2007) 047 [hep-ph/0611326] [INSPIRE].

[30] W. Porod, SPheno, a program for calculating supersymmetric spectra, SUSY particle decays and SUSY particle production at $e^{+} e^{-}$colliders, Comput. Phys. Commun. 153 (2003) 275 [hep-ph/0301101] [INSPIRE].

[31] W. Porod and F. Staub, SPheno 3.1: extensions including flavour, CP-phases and models beyond the MSSM, Comput. Phys. Commun. 183 (2012) 2458 [arXiv:1104.1573] [inSPIRE].

[32] H.E. Haber and R. Hempfling, The renormalization group improved Higgs sector of the minimal supersymmetric model, Phys. Rev. D 48 (1993) 4280 [hep-ph/9307201] [InSPIRE].

[33] J.R. Ellis, G. Ridolfi and F. Zwirner, Radiative corrections to the masses of supersymmetric Higgs bosons, Phys. Lett. B 257 (1991) 83 [INSPIRE].

[34] J.R. Ellis, G. Ridolfi and F. Zwirner, On radiative corrections to supersymmetric Higgs boson masses and their implications for LEP searches, Phys. Lett. B 262 (1991) 477 [INSPIRE].

[35] F. Gabbiani, E. Gabrielli, A. Masiero and L. Silvestrini, A complete analysis of FCNC and CP constraints in general SUSY extensions of the Standard Model, Nucl. Phys. B 477 (1996) 321 [hep-ph/9604387] [INSPIRE].

[36] M. Misiak, S. Pokorski and J. Rosiek, Supersymmetry and FCNC effects, Adv. Ser. Direct. High Energy Phys. 15 (1998) 795 [hep-ph/9703442] [INSPIRE].

[37] A. Behring, C. Gross, G. Hiller and S. Schacht, Squark flavor implications from $B \rightarrow K^{*} \ell^{+} \ell^{-}$, JHEP 08 (2012) 152 [arXiv: 1205.1500] [INSPIRE].

[38] G. Colangelo and G. Isidori, Supersymmetric contributions to rare kaon decays: beyond the single mass insertion approximation, JHEP 09 (1998) 009 [hep-ph/9808487] [INSPIRE].

[39] W. Altmannshofer, A.J. Buras, D.M. Straub and M. Wick, New strategies for new physics search in $B \rightarrow K^{*} \nu \bar{\nu}, B \rightarrow K \nu \bar{\nu}$ and $B \rightarrow X_{s} \nu \bar{\nu}$ decays, JHEP 04 (2009) 022 [arXiv: 0902.0160] [INSPIRE].

[40] CMS collaboration, Combined multilepton and diphoton limit on $t \rightarrow c H$, CMS-PAS-HIG-13-034, CERN, Geneva Switzerland (2013).

[41] J. Guasch and J. Solà, FCNC top quark decays: a door to SUSY physics in high luminosity colliders?, Nucl. Phys. B 562 (1999) 3 [hep-ph/9906268] [INSPIRE].

[42] J.J. Cao et al., SUSY-induced FCNC top-quark processes at the Large Hadron Collider, Phys. Rev. D 75 (2007) 075021 [hep-ph/0702264] [INSPIRE]. 
[43] A. Crivellin et al., SUSY_FLAVOR v2: a computational tool for FCNC and CP-violating processes in the MSSM, Comput. Phys. Commun. 184 (2013) 1004 [arXiv:1203.5023] [INSPIRE].

[44] Electroweak radiative $B$ decays webpage, http://www.slac.stanford.edu/xorg/hfag/rare/2012/radll/index.html.

[45] LHCb collaboration, Measurement of the $B_{s}^{0} \rightarrow \mu^{+} \mu^{-}$branching fraction and search for $B^{0} \rightarrow \mu^{+} \mu^{-}$decays at the LHCb experiment, Phys. Rev. Lett. 111 (2013) 101805 [arXiv: 1307.5024] [INSPIRE].

[46] CMS collaboration, Measurement of the $B_{s} \rightarrow \mu^{+} \mu^{-}$branching fraction and search for $B^{0} \rightarrow \mu^{+} \mu^{-}$with the CMS experiment, Phys. Rev. Lett. 111 (2013) 101804 [arXiv: 1307.5025] [INSPIRE].

[47] A. Crivellin and U. Nierste, Supersymmetric renormalisation of the CKM matrix and new constraints on the squark mass matrices, Phys. Rev. D 79 (2009) 035018 [arXiv:0810.1613] [INSPIRE].

[48] A. Crivellin, Effects of right-handed charged currents on the determinations of $\left|V_{u b}\right|$ and $\left|V_{c b}\right|$, Phys. Rev. D 81 (2010) 031301 [arXiv:0907.2461] [INSPIRE].

[49] A. Crivellin, L. Hofer and J. Rosiek, Complete resummation of chirally-enhanced loop-effects in the MSSM with non-minimal sources of flavor-violation, JHEP 07 (2011) 017 [arXiv:1103.4272] [INSPIRE].

[50] J.M. Frere, D.R.T. Jones and S. Raby, Fermion masses and induction of the weak scale by supergravity, Nucl. Phys. B 222 (1983) 11 [INSPIRE].

[51] L. Álvarez-Gaumé, J. Polchinski and M.B. Wise, Minimal low-energy supergravity, Nucl. Phys. B 221 (1983) 495 [INSPIRE].

[52] J.P. Derendinger and C.A. Savoy, Quantum effects and $\mathrm{SU}(2) \times \mathrm{U}(1)$ breaking in supergravity gauge theories, Nucl. Phys. B 237 (1984) 307 [INSPIRE].

[53] C. Kounnas, A.B. Lahanas, D.V. Nanopoulos and M. Quirós, Low-energy behavior of realistic locally supersymmetric Grand Unified Theories, Nucl. Phys. B 236 (1984) 438 [InSPIRE].

[54] J.A. Casas and S. Dimopoulos, Stability bounds on flavor violating trilinear soft terms in the MSSM, Phys. Lett. B 387 (1996) 107 [hep-ph/9606237] [INSPIRE].

[55] F. Borzumati, G.R. Farrar, N. Polonsky and S.D. Thomas, Soft Yukawa couplings in supersymmetric theories, Nucl. Phys. B 555 (1999) 53 [hep-ph/9902443] [INSPIRE].

[56] J.-H. Park, Metastability bounds on flavour-violating trilinear soft terms in the MSSM, Phys. Rev. D 83 (2011) 055015 [arXiv: 1011.4939] [INSPIRE].

[57] S.P. Martin and M.T. Vaughn, Two loop renormalization group equations for soft supersymmetry breaking couplings, Phys. Rev. D 50 (1994) 2282 [Erratum ibid. D 78 (2008) 039903] [hep-ph/9311340] [INSPIRE].

[58] BeLLE collaboration, I. Adachi et al., Evidence for $B^{-} \rightarrow \tau^{-} \bar{\nu}_{\tau}$ with a hadronic tagging method using the full data sample of Belle, Phys. Rev. Lett. 110 (2013) 131801 [arXiv: 1208.4678] [INSPIRE].

[59] F. Feroz, M.P. Hobson and M. Bridges, MultiNest: an efficient and robust Bayesian inference tool for cosmology and particle physics, Mon. Not. Roy. Astron. Soc. 398 (2009) 1601 [arXiv:0809.3437] [INSPIRE]. 
[60] P. Gondolo et al., DarkSUSY: computing supersymmetric dark matter properties numerically, JCAP 07 (2004) 008 [astro-ph/0406204] [INSPIRE].

[61] P. Bechtle, O. Brein, S. Heinemeyer, G. Weiglein and K.E. Williams, HiggsBounds: confronting arbitrary Higgs sectors with exclusion bounds from LEP and the Tevatron, Comput. Phys. Commun. 181 (2010) 138 [arXiv:0811.4169] [InSPIRE].

[62] P. Bechtle, O. Brein, S. Heinemeyer, G. Weiglein and K.E. Williams, HiggsBounds 2.0.0: confronting neutral and charged Higgs sector predictions with exclusion bounds from LEP and the Tevatron, Comput. Phys. Commun. 182 (2011) 2605 [arXiv:1102.1898] [INSPIRE].

[63] P. Bechtle et al., HiggsBounds 4: improved tests of extended Higgs sectors against exclusion bounds from LEP, the Tevatron and the LHC, Eur. Phys. J. C 74 (2014) 2693 [arXiv: 1311.0055] [INSPIRE].

[64] P. Bechtle, S. Heinemeyer, O. Stål, T. Stefaniak and G. Weiglein, HiggsSignals: confronting arbitrary Higgs sectors with measurements at the Tevatron and the LHC, Eur. Phys. J. C 74 (2014) 2711 [arXiv: 1305.1933] [INSPIRE].

[65] C. Bobeth, T. Ewerth, F. Krüger and J. Urban, Analysis of neutral Higgs boson contributions to the decays $B_{s} \rightarrow \ell^{+} \ell^{-}$and $\bar{B} \rightarrow K \ell^{+} \ell^{-}$, Phys. Rev. D 64 (2001) 074014 [hep-ph/0104284] [INSPIRE].

[66] LUX collaboration, D.S. Akerib et al., First results from the LUX dark matter experiment at the Sanford Underground Research Facility, Phys. Rev. Lett. 112 (2014) 091303 [arXiv: 1310.8214] [INSPIRE].

[67] XENON1T collaboration, E. Aprile, The XENON1T dark matter search experiment, Springer Proc. Phys. 148 (2013) 93 [arXiv:1206.6288] [INSPIRE].

[68] M. Blanke, G.F. Giudice, P. Paradisi, G. Perez and J. Zupan, Flavoured naturalness, JHEP 06 (2013) 022 [arXiv: 1302.7232] [INSPIRE].

[69] S.R. Coleman and E.J. Weinberg, Radiative corrections as the origin of spontaneous symmetry breaking, Phys. Rev. D 7 (1973) 1888 [INSPIRE]. 